ARTICLE

DOI: $10.1038 / s 41467-018-06409-5$

\title{
Dopamine-endocannabinoid interactions mediate spike-timing-dependent potentiation in the striatum
}

Hao Xu ${ }^{1,2}$, Sylvie Perez ${ }^{1,2}$, Amandine Cornil $^{3}$, Bérangère Detraux ${ }^{3}$, llya Prokin ${ }^{4,5}$, Yihui Cui ${ }^{1,2}$, Bertrand Degos ${ }^{1,2}$, Hugues Berry (1) 4,5, Alban de Kerchove d'Exaerde (i) ${ }^{3,6}$ \& Laurent Venance (1) ${ }^{1,2}$

Dopamine modulates striatal synaptic plasticity, a key substrate for action selection and procedural learning. Thus, characterizing the repertoire of activity-dependent plasticity in striatum and its dependence on dopamine is of crucial importance. We recently unraveled a striatal spike-timing-dependent long-term potentiation (tLTP) mediated by endocannabinoids (eCBs) and induced with few spikes ( 5-15). Whether this eCB-tLTP interacts with the dopaminergic system remains to be investigated. Here, we report that eCB-tLTP is impaired in a rodent model of Parkinson's disease and rescued by L-DOPA. Dopamine controls eCBtLTP via dopamine type-2 receptors $\left(D_{2} R\right)$ located presynaptically in cortical terminals. Dopamine-endocannabinoid interactions via $D_{2} R$ are required for the emergence of tLTP in response to few coincident pre- and post-synaptic spikes and control eCB-plasticity by modulating the long-term potentiation (LTP)/depression (LTD) thresholds. While usually considered as a depressing synaptic function, our results show that eCBs in the presence of dopamine constitute a versatile system underlying bidirectional plasticity implicated in basal ganglia pathophysiology.

\footnotetext{
${ }^{1}$ Center for Interdisciplinary Research in Biology, College de France, INSERM U1050, CNRS UMR 7241, Labex Memolife, 75005 Paris, France. ${ }^{2}$ University Pierre et Marie Curie, ED 158, Paris Sciences et Lettres, 75005 Paris, France. ${ }^{3}$ Laboratory of Neurophysiology, Université Libre de Bruxelles, ULB Neuroscience Institute, Brussels 1070, Belgium. ${ }^{4}$ INRIA, Villeurbanne 69603, France. ${ }^{5}$ University of Lyon, LIRIS UMR 5205, Villeurbanne 69621, France.

${ }^{6}$ WELBIO, Wavre 1300, Belgium. Correspondence and requests for materials should be addressed to L.V. (email: laurent.venance@college-de-france.fr)
} 
E ndocannabinoids (eCBs) have emerged as a major signaling system in learning and memory because of their powerful influence on synaptic plasticity, mainly as depressing synaptic function ${ }^{1,2}$. There exists a growing body of evidence that eCBs are also associated with synaptic potentiation ${ }^{2}$. Indeed, eCBs promote long-term potentiation (LTP) at mixed (chemical and electrical) synapses of the goldfish Mauthner cell via intermediary dopaminergic neurons ${ }^{3}$ or at hippocampal CA1 synapses via a $\mathrm{GABA}_{\mathrm{A}}$ receptor-mediated mechanism ${ }^{4-6}$, facilitation of hippocampal LTP via eCB-induced presynaptic depression of GABAergic transmission ${ }^{7}$, and heterosynaptic short-term potentiation via the astrocytic network ${ }^{8}$. Recently, a direct role of $\mathrm{eCBs}$ has been found in promoting LTP in the dentate gyrus $^{9,10}$, the somatosensory cortex ${ }^{11}$, and the dorsolateral striatum $^{12,13}$.

The striatum is a strategic gate extracting pertinent cortical information and a major site of memory formation. Indeed, synaptic plasticity at corticostriatal synapses ${ }^{14-19}$, changes of striatal neuronal activity ${ }^{14,20}$, and corticostriatal coherence ${ }^{21}$ have been associated with the acquisition or extinction in the behavioral repertoire. The striatum receives a dense innervation from midbrain dopaminergic neurons, one of the key players of basal ganglia function in action selection and associative learning 22 . In particular, dopamine plays a crucial role in goal-directed behavior and reinforcement learning, which is dramatically highlighted in neuronal disorders affecting corticostriatal information processing, such as Parkinson's disease ${ }^{23}$.

Dopamine and synaptic plasticity signaling pathways interaction is required for the induction of the main form of striatal plasticity: the endocannabinoid-mediated long-term depression (eCB-LTD $)^{24}$. At corticostriatal synapses, eCB-LTD has consistently been observed using various cell-conditioning paradigms (high- and low-frequency stimulation, theta-burst protocols, or spike-timing-dependent plasticity ${ }^{24-26}$. The striatum receives a wide range of patterns of cortical activities from isolated trains of few spikes to prolonged bursting events. While plasticity under prolonged activation is well elucidated, its expression in response to few spikes remains less documented. To this end, we chose spike-timing-dependent plasticity (STDP) as a synaptic Hebbian learning paradigm ${ }^{27}$. Indeed, STDP (tLTP and tLTD) depends on the relative timing between pre- and postsynaptic spikes, and relies on much fewer events (around 100 paired stimulations) than the high- or low-frequency stimulation protocols (hundreds of stimulations). In the striatum, bidirectional STDP with NMDAR-mediated tLTP and eCB-mediated tLTD, has been reported with $100-150$ paired stimulations ${ }^{26,28-32}$. Using STDP, we recently reported a new form of plasticity in the dorsolateral striatum: a striatal spike-timing-dependent potentiation (tLTP) induced by few coincident pre- and post-synaptic spikes ( $\sim 5-15)$, mediated by eCBs (eCB-tLTP) through a signaling pathway that relies on the activation of type-1 cannabinoid receptor $\left(\mathrm{CB}_{1} \mathrm{R}\right)$ and transient receptor potential vanilloid type-1 (TRPV1) and on eCB dynamics ${ }^{12,13}$. Corticostriatal eCB-tLTP relies on postsynaptic synthesis and release of eCBs, which mainly activate presynaptic $C_{1} R$, and displays a presynaptic locus of plasticity maintenance. We previously showed that the bidirectionality of eCB-dependent STDP in striatum is controlled by eCB-levels: prolonged and moderate release of eCBs leads to eCB-tLTD, whereas brief and large eCB transients produce eCB-tLTP ${ }^{13}$. Moreover, in striatum, eCB-STDP is controlled by the protein kinase A (PKA) and calcineurin activity balance, such as eCBtLTD requires active calcineurin whereas eCB-tLTP necessitates the activity of presynaptic PKA ${ }^{12,13}$.

Thus, eCBs not only promote depression but also potentiation, i.e., they act as a bidirectional system, depending on the regime of activity pattern on either side of the synapse. The interactions between the dopaminergic system and eCB-LTD have thoroughly been studied and a solid experimental support exists for the efficient modulation of eCB-LTD by dopamine ${ }^{24}$. However, whether and how eCB-tLTP may be modulated by dopamine and consequently be affected in Parkinson's disease remains unknown. Thus, considering the crucial role of dopamine in striatal physiology and pathophysiology and in particular in synaptic plasticity $22-24,33,34$, we question here the implication of dopamine in the expression of eCB-tLTP. We observe that eCBtLTP is impaired in a rodent model of Parkinson's disease and is rescued by L-DOPA treatment. We find that opto-inhibition of dopaminergic neurons prevent eCB-tLTP and that dopamine type-2 receptors $\left(D_{2} R\right)$ on cortical terminals are required for eCBtLTP expression. We provide a biologically plausible mathematical model for the dynamics of the implicated signaling pathways. Combining our experimental results and modeling, we show that dopamine controls not only the induction but also the polarity via presynaptic $\mathrm{D}_{2} \mathrm{R}$ (tLTP vs tLTD) of eCB-plasticity by modulating the effective eCB thresholds.

\section{Results}

Distinct STDP activity patterns induced eCB-tLTP and -tLTD. STDP is a synaptic Hebbian learning rule in which synaptic weight changes depend on the activity on both sides of the synapse $^{27}$. Corticostriatal synapses exhibit a bidirectional eCBdependent STDP in which eCB-tLTD ${ }^{12,28-32}$ or eCB-tLTP ${ }^{12,13}$ are induced depending on the spike timing $\left(\Delta t_{\mathrm{STDP}}\right)$ and on the number of pairings $\left(N_{\text {pairings }}\right)$. In the dorsolateral striatum, we recently reported that a low number of pairings $\left(N_{\text {pairings }}=5-15\right)$ induces an eCB-tLTP, dependent on the activation of $\mathrm{CB}_{1} \mathrm{R}$ and TRPV1 ${ }^{12,13}$. It is well documented that eCB-LTD (mainly induced with high- or low-frequency stimulation protocols) is controlled by dopamine levels ${ }^{24}$. However, the dopaminergic control of eCB-tLTP remains undocumented. Here, to examine the dopamine dependence of eCB-tLTP, we carried out whole-cell recordings from medium-sized spiny neurons (MSNs) of the dorsolateral striatum (Fig. 1a): after measuring baseline EPSCs for $10 \mathrm{~min}$, recordings were switched to current-clamp to pair a single EPSP induced by presynaptic cortical stimulation with a single postsynaptic spike induced by a brief depolarization of the MSN (Fig. 1a). The STDP protocol consisted in pairing of preand postsynaptic stimulations separated by a certain fixed timing interval $\left(\Delta t_{\text {STDP }}\right)$ and repeated 10 or 100 times at $1 \mathrm{~Hz} . \Delta t_{\text {STDP }}<0$ when the post-synaptic stimulation occurs before the paired presynaptic one (post-pre pairings) (Fig. 1a), whereas $\Delta t_{\text {STDP }}>0$ when the pre-synaptic stimulation occurs before the postsynaptic one (pre-post pairings) (Fig. S1a).

We observed that 10 post-pre and 100 pre-post pairings induced a bidirectional anti-Hebbian eCB-STDP with eCB-tLTP induced by 10 post-pre pairings (Fig. 1b, c) and eCB-tLTD by 100 pre-post pairings (Supplementary Fig. 1). GABA operates as a Hebbian/anti-Hebbian switch at corticostriatal synapses ${ }^{32,35}$ because corticostriatal STDP polarity depends on the presence (ex vivo Hebbian STDP ${ }^{28,29}$ ) or the absence of $\mathrm{GABA}_{\mathrm{A}}$ receptor antagonists (ex vivo anti-Hebbian STDP12,13,30,31, as well as in this study; in vivo anti-Hebbian STDP ${ }^{36}$ ). We thus recorded STDP in the absence of $\mathrm{GABA}_{\mathrm{A}} \mathrm{R}$ antagonist to preserve the local striatal microcircuits and the anti-Hebbian polarity as observed in vivo ${ }^{36}$. Figure $1 \mathrm{~b}$ and Supplementary Figure $1 \mathrm{~b}$ show examples of tLTP and tLTD induced by 10 post-pre and 100 pre-post pairings, respectively. To summarize, 10 post-pre STDP pairings $\left(-30<\Delta t_{\text {STDP }}<0 \mathrm{~ms}\right.$ ) induced tLTP (mean value of the EPSC amplitude recorded $50 \mathrm{~min}$ after STDP protocol: $158 \pm 11 \%, p=$ $0.0006, n=9)$, which was prevented by AM251 $(3 \mu \mathrm{M})$, a $\mathrm{CB}_{1} \mathrm{R}$ specific inhibitor $(80 \pm 14 \%, p=0.2223, n=5)$ or with AMG9810 
a

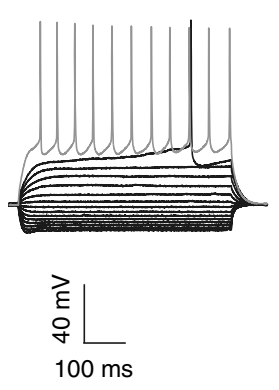

d

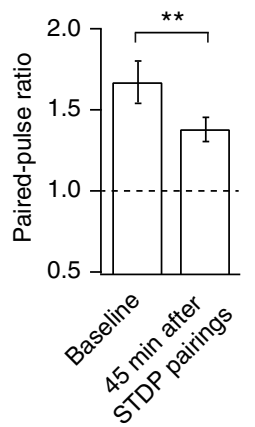

b

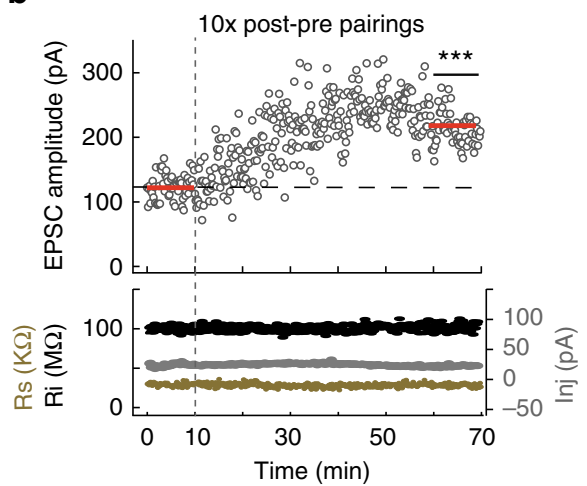

f

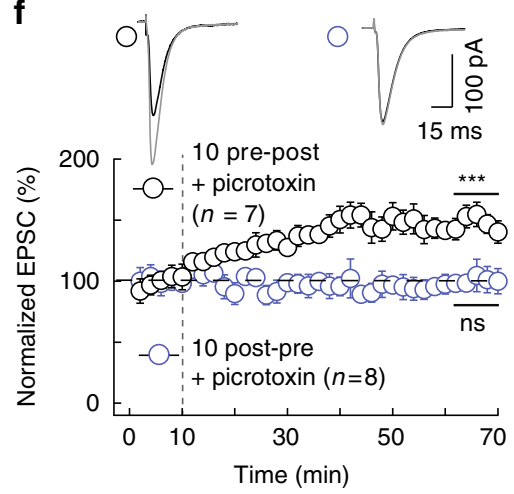

C
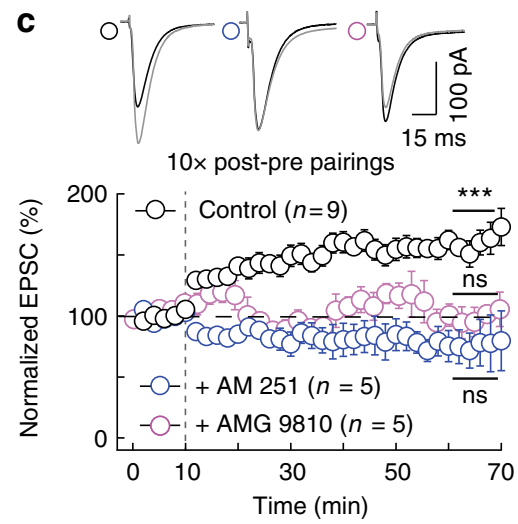

g

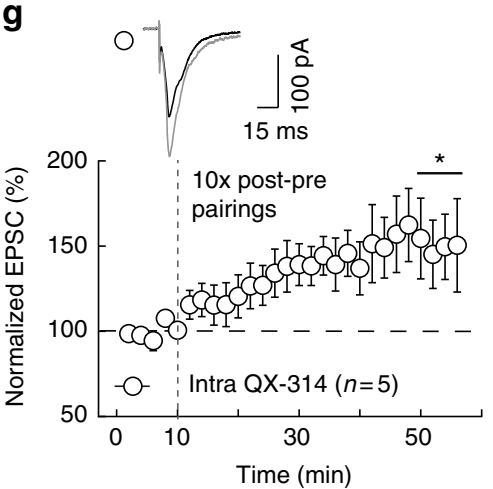

Fig. 1 eCB-tLTP is induced by low number of pairings. a Characteristic voltage responses of a MSN to a series of 500 ms current pulses from -150 to $+180 \mathrm{pA}$ with current steps increasing by $30 \mathrm{pA}$ (black traces) and to $+60 \mathrm{pA}$ above spike threshold (grey trace). STDP protocol: a spike-evoked in one MSN was paired with a cortical stimulation repeated 10 times at $1 \mathrm{~Hz} . \Delta t_{S T D P}$ indicates the temporal time shift between pre- and postsynaptic stimulations. b, c 10 post-pre pairings induced $t L T P C_{1} R$ - and TRPV1-activation dependent. b Example of tLTP induced by 10 post-pre pairings ( $\Delta t_{S T D P}=-13$ ms). Top, EPSC strength before and after 10 pairings $(124 \pm 4 \mathrm{pA}$ and $203 \pm 3 \mathrm{pA}, p<0.0001)$. Bottom, time courses of input resistance $\left(R_{i}\right)($ before, $99 \pm 1 \mathrm{M} \Omega$; after, $105 \pm 1 \mathrm{M} \Omega$; change of $6 \%$ ), access resistance $\left(R_{\text {access }}\right)$ (before, $30 \pm 1 \mathrm{k} \Omega$; after, $29 \pm 1 \mathrm{k} \Omega$; change of $3 \%$ ) and injected current $\left(I_{\text {inj }}\right)$ (before, $24 \pm 0.3 \mathrm{pA}$; after, $23 \pm 0.2 \mathrm{pA})$. c Summary of STDP experiments showing that eCB-tLTP induced with 10 post-pre pairings $(n=9,9$ cells out of 9 resulted in tLTP) is prevented by a specific $C_{1} R$ or TRPV1 inhibitor, AM251 ( $3 \mu \mathrm{m}, n=5,1 / 5$ showed tLTP) and AMG9810 $(1 \mu M, n=5,0 / 5$ showed tLTP), respectively. d Summary bar graphs ( $n=16 \mathrm{MSNs}$ ) of paired-pulse cortical stimulations (50 ms interstimulus interval) illustrate a decrease of facilitation after 10 postpre pairings. This indicates a presynaptic locus of the eCB-tLTP. e Mean variance analysis $\left(\mathrm{CV}^{-2}\right.$ plasticity/baseline, $\left.n=21\right)$ during baseline and 45 min after STDP pairings indicates a presynaptic locus of the eCB-tLTP. $\mathbf{f}$ With inhibition of ionotropic GABAergic transmission (picrotoxin $50 \mu M$ ), tLTP was observed with pre-post ( $n=7,6 / 7$ showed tLTP) but not with post-pre $(n=8,1 / 8$ showed tLTP) pairings. GABA controls the time dependence but not the magnitude of eCB-tLTP. $\mathbf{g}$ Intracellular application of a Na+ channel blocker, QX-314 ( $1 \mu \mathrm{m}, n=5,5 / 5$ showed tLTP), did not prevent 10 post-pre pairings LTP. Representative traces are the average of 15 EPSCs during baseline (black traces) and 45 min after STDP protocol (grey traces). Vertical grey dashed line indicates the STDP protocol. Error bars represent sem. ${ }^{\star} p<0.05 ;{ }^{\star \star} p<0.01$; ${ }^{\star \star \star} p<0.001$; ns: not significant by $t$-test, two-tailed (b), one sample $t$-test $(\mathbf{c}, \mathbf{f}, \mathbf{g})$ or Wilcoxon Signed rank test $(\mathbf{c}, \mathbf{d})$

$(1 \mu \mathrm{M})$, a TRPV1-specific inhibitor $(98 \pm 7 \%, p=0.7723, n=5)$ (Fig. 1c) as recently reported ${ }^{12,13} .100$ pre-post pairings $(0<$ $\left.\Delta t_{\mathrm{STDP}}<+30 \mathrm{~ms}\right)$ induced tLTD $(71 \pm 10 \%, p=0.0253, n=7)$, which was prevented by AM251 $(103 \pm 7 \%, p=0.7500, n=7)$ or with AMG9810 $(99 \pm 10 \%, p=0.9197, n=5)$ (Supplementary Fig. $1 c-d)$ confirming previous reports ${ }^{13,28-32}$. Thus, we confirm that eCB system encodes for bidirectional striatal plasticity depending on the number and order of pairings.

$\mathrm{CB}_{1} \mathrm{R}$ being located at the presynaptic terminals of the corticostriatal pathway ${ }^{1,24}$, the locus of eCB-tLTP maintenance would likely be presynaptic. We applied presynaptic paired-pulses (50 $\mathrm{ms}$ interpulse interval) before and $45 \mathrm{~min}$ after STDP pairings, and observed a EPSC paired-pulse facilitation ${ }^{12,13}$. We observed a decrease of the paired-pulse ratio (paired-pulse ratio $\left._{\text {plasticity/baseline }}=0.855 \pm 0.04, \quad p=0.0032, \quad n=16\right) \quad 45 \mathrm{~min}$ after the 10 post-pre pairings (Fig. 1d), which indicates a presynaptic locus of eCB-tLTP. The mean variance analysis of EPSCs gave a $\mathrm{CV}^{-2}$ value of $3.55 \pm 0.60(p<0.0001, n=21)$, which also indicates a presynaptic locus for eCB-tLTP (Fig. 1e).
We investigated the impact of GABAergic networks on eCBtLTP, which could arise from a decrease of GABA release, as reported for hippocampal eCB-LTP ${ }^{4,7}$. When ionotropic GABAergic transmission was blocked with picrotoxin $(50 \mu \mathrm{M})$, tLTP was observed for 10 pre-post pairings $(142 \pm 18 \%, p=$ $0.0008, n=7)$, whereas no detectable plasticity was found for 10 post-pre pairings $(99 \pm 19 \%, p=0.8909, n=8)$ (Fig. 1f). tLTP magnitudes observed in control and picrotoxin conditions were not significantly different $(p=0.4575)$. Therefore, GABAergic circuits do not control the expression of eCB-tLTP but control its timing dependence, in agreement with the GABA effects reported for 100 pairings NMDA-tLTP and eCB-LTD ${ }^{32,35}$.

The subthreshold postsynaptic depolarization itself is a key factor in the induction of striatal plasticity ${ }^{37}$. We tested whether postsynaptic spikes were required for eCB-tLTP induction. To this aim, we delivered intracellularly in the postsynaptic recorded MSN via the patch pipette the QX-314 $(10 \mu \mathrm{M})$, a Na ${ }^{+}$channel inhibitor, and observed tLTP with 10 post-pre pairings (158 \pm 21\%, $p=0.0483, n=5$; Fig. 1f). Therefore, subthreshold 
depolarizations (30 ms duration) appeared sufficient to induce striatal eCB-tLTP.

eCB-tLTP is impaired in Parkinson's and rescued by L-DOPA. Whereas NMDAR-LTP and eCB-LTD striatal synaptic plasticity are dependent on dopamine and therefore are impacted in Parkinson's disease $23,24,34$, it is not known whether eCB-tLTP is affected in Parkinson's disease. To address this question, we used a rat model of Parkinson's disease in which dopaminergic transmission is impaired. We performed unilateral lesion of the substantia nigra pars compacta $(\mathrm{SNc})$ with 6-hydroxy-dopamine (6-OHDA), a neurotoxic synthetic organic compound which leads, when associated with desipramine, to the selective degeneration of dopaminergic neurons (Fig. 2a, b). We observed a massive degeneration of nigral dopaminergic neurons and of their striatal terminals as shown by the dramatic decrease of striatal tyrosine hydroxylase (TH) staining by $64 \pm 5 \%(p<0.0001, n=7)$ 2 weeks after the 6-OHDA lesions at $\mathrm{P}_{50}$ (Fig. 2b). We also used sham-operated animals with saline injection for comparison with the 6-OHDA-lesioned rats (Fig. 2a, b). We first verified that
tLTP was induced with 10 post-pre pairings in adult control rats (i.e., without any surgery and recorded at similar age than the sham-operated and 6-OHDA-lesioned rats, $\left.\mathrm{P}_{(60-65)}\right)(164 \pm 16 \%$, $p=0.0034, n=10$; Supplementary Fig. 2a) and in sham-operated rats $(147 \pm 7 \%, p=0.0002, n=9$; Fig. $2 \mathrm{c})$. The tLTP induced in adult control animals and in sham-operated rats were not different $(p=0.7019)$. In contrast, in the 6-OHDA-lesioned animals, 10 post-pre pairings failed to induce any plasticity $(94 \pm 5 \%$, $p=0.2741, n=9$; Fig. $2 \mathrm{c}$ ) thus showing that the degeneration of dopaminergic neurons is deleterious for the induction of eCB-tLTP.

We next tested whether treatment with L-3,4-dihydroxyphenylalanine (L-DOPA; Fig. 2a), a mainstay for symptomatic treatment of Parkinson's disease, could rescue eCB-tLTP in 6-OHDAlesioned rats. In slices obtained from 6-OHDA-lesioned animals treated with L-DOPA, 10 post-pre pairings caused tLTP (171 \pm $19 \%, p=0.0096, n=7$; Fig. $2 \mathrm{~d}$ ); this tLTP was not different from the tLTP obtained in sham-operated rats $(p=0.3481)$. tLTP was also observed in the sham-operated animals L-DOPA-treated (164 $\pm 20 \%, p=0.0201, n=7$; Supplementary Fig. $2 b)$ and this

a

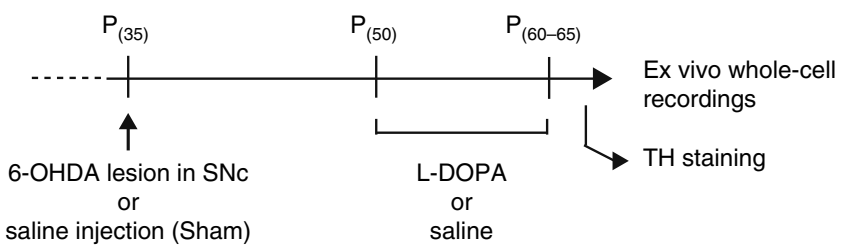

$\left\{\begin{array}{l}\text { - 6-OHDA + L-DOPA } \\ \text {-6-OHDA + saline } \\ \text { - Sham + L-DOPA } \\ \text { - Sham + saline }\end{array}\right.$

b
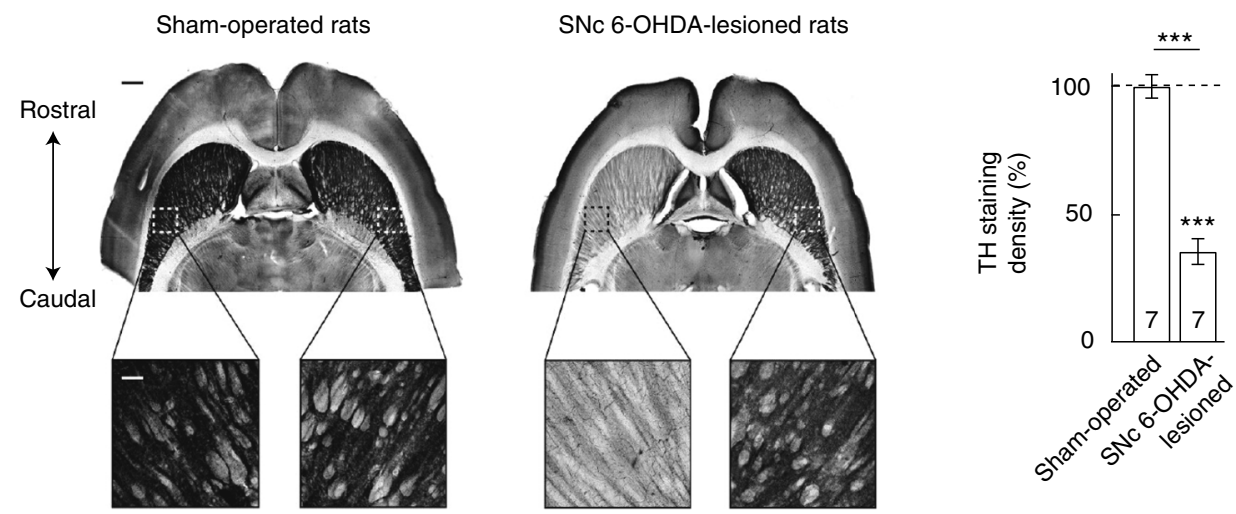

C

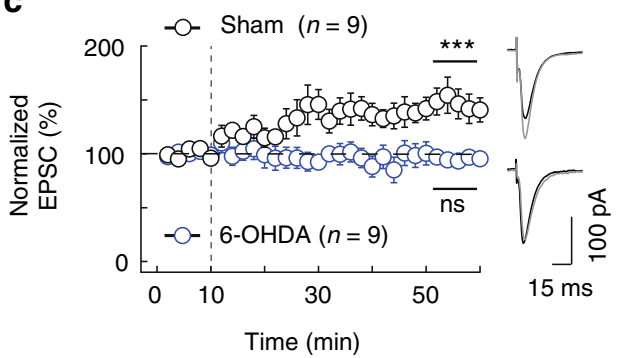

d

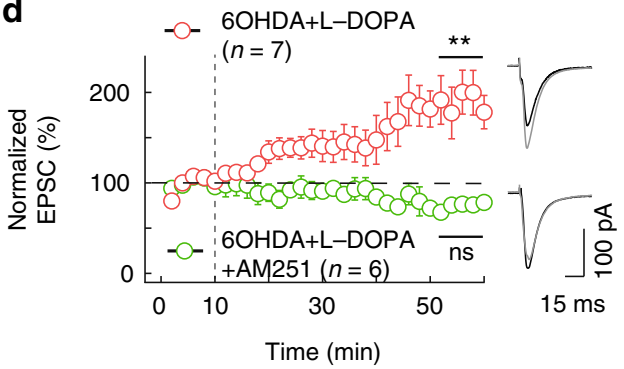

Fig. 2 eCB-tLTP is impaired in a rat model of Parkinson's and rescued by L-DOPA. a Protocols of the 6-OHDA lesion (or sham) in $P_{35}$ rats followed 2 weeks after by chronic L-DOPA treatment (or saline) for 10 days. b Unilateral 6-OHDA injection in SNc led to degeneration of dopaminergic nigral neurons and a loss of their striatal afferences as illustrating by TH immunostaining in horizontal brain slices. Note that sham-operated rats display equivalent TH staining in both striata. Scale bars: $1 \mathrm{~mm}$ and $200 \mu \mathrm{m}$. Right panel: summary bar graph of TH staining quantification. c 10 post-pre pairings induced eCB-tLTP in sham-operated rats $(n=9,9 / 9$ cells showed tLTP) while no plasticity was observed in 6-OHDA-lesioned rats $(n=9,1 / 9$ cells showed tLTP). $\mathbf{d}$ Chronic L-DOPA treatment consisting in twice daily injection of L-DOPA $(10 \mathrm{mg} / \mathrm{kg})$ for 10 days, 2 weeks after $6-\mathrm{OHDA}$ lesion allowed to recover tLTP $(n=7,6 / 7$ cells showed tLTP) induced with 10 post-pre pairings. This $L L T P$ was $C B_{1} R$-mediated since prevented by $A M 251$ ( $3 \mu \mathrm{m}, n=6,0 / 6$ cells showed tLTP) in 6-OHDA-lesioned rats treated with L-DOPA. Representative traces are the average of 15 EPSCs during baseline (black traces) and 45 min after STDP protocol (grey traces). Vertical grey dashed line indicates the STDP protocol. Error bars represent sem. ${ }^{\star \star} p<0.01$; ${ }^{\star \star \star} p<0.001$; ns: not significant by one sample $t$-test $(\mathbf{c}, \mathbf{d})$ or Wilcoxon Signed rank test $(\mathbf{b})$ 
tLTP was not different from the one obtained in sham-operated rats $(p=0.4038)$ or in 6-OHDA-lesioned animals treated with L-DOPA $(p=0.7791)$. In 6-OHDA-lesioned animals L-DOPAtreated, tLTP induced with 10 post-pre pairings was $\mathrm{CB}_{1} \mathrm{R}$ mediated since prevented by AM251 $(81 \pm 6 \%, p=0.0244, n=6$; Fig. 2d). Therefore, eCB-tLTP was impaired in a rodent model of Parkinson's disease and was rescued by L-DOPA treatment.

Dopamine is required for eCB-tLTP induction. Cortical stimulation enhances dopamine release in the dorsal striatum ${ }^{38-40}$. We investigated if dopamine was required during the few pairings of the induction phase of eCB-tLTP. To this intent, we optoinhibited dopaminergic neurons during the few pairings responsible for eCB-tLTP in DAT-Cre ${ }^{+/-}:$A Arch3-GFP $P^{+/-}$mice. We confirmed that Arch3-GFP expression was restricted to dopaminergic neurons and terminals in the striatum with $\mathrm{TH}$ and GFP immunostainings $(n=10)$ in $\mathrm{SNc}$ and dorsal striatum (Fig. 3a). We ensured the efficiency of the opto-inhibition by cellattached recordings of the spontaneous spiking activity of dopaminergic neurons in the SNc from DAT-Cre ${ }^{+/-}:$:Arch3-GFP ${ }^{+/-}$ mice. Upon photostimulation, we observed an absence of spikes $(2.1 \pm 0.5 \mathrm{~Hz}$ without light and $0 \mathrm{~Hz}$ with light, $n=5$; with $I_{\mathrm{inj}}=0 \mathrm{pA}$ ) (Fig. 3b) and a hyperpolarization of the resting
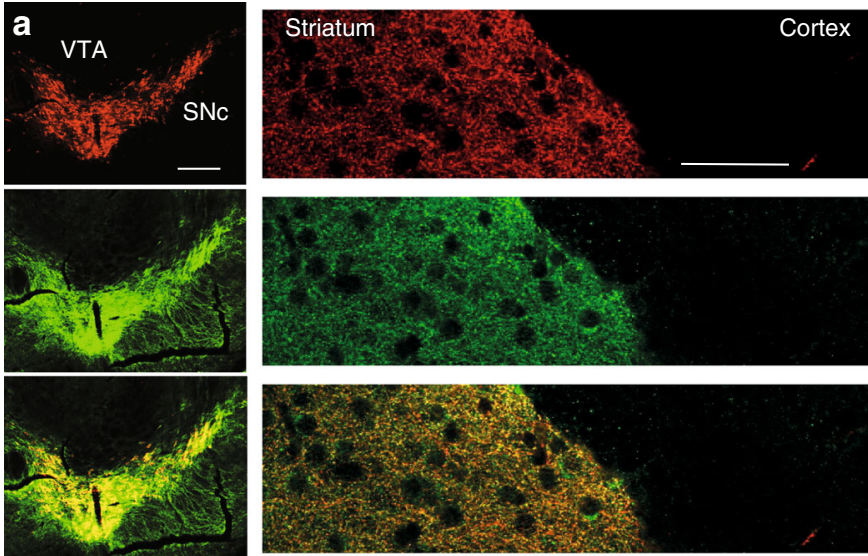

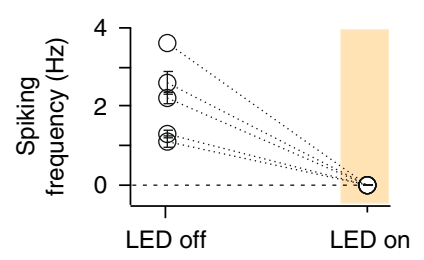

b

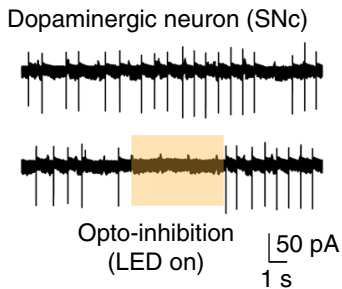

d

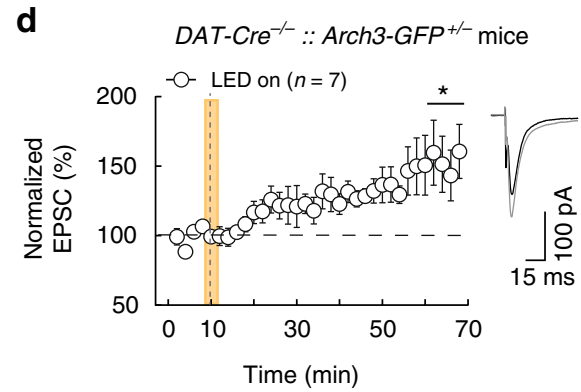

$\mathbf{f}$ e

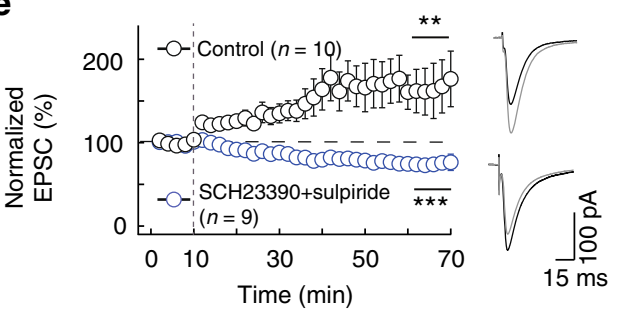

C

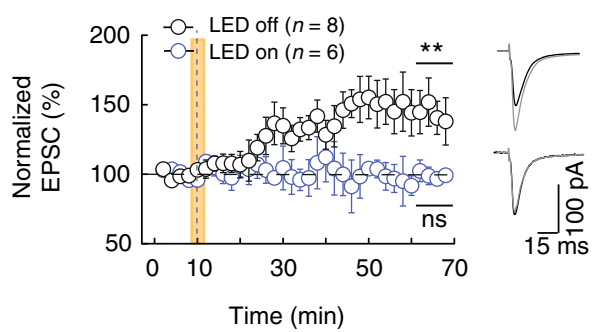

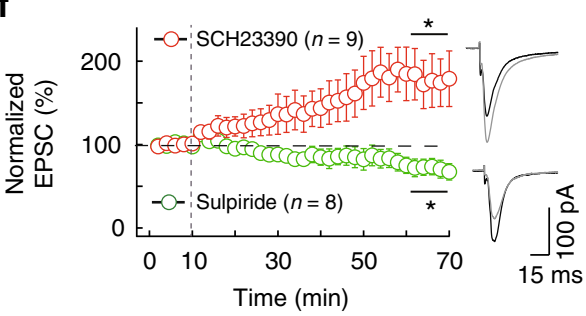

Fig. 3 eCB-LTP requires dopamine release during pairings and is $D_{2} R$-dependent. a-d Induction of eCB-LTP requires dopamine release during the STDP paired-activity paradigm. a Double immunostaining for tyrosine hydroxylase (indicating dopaminergic cell bodies in SNc and dopaminergic afferences in striatum; upper panels) and GFP (indicating Arch3-expression; middle panels) in SNc (left panels) and in striatum (right panels); merge images are shown in the lower panels. Scale bars: $500 \mu \mathrm{m}$ (SNc) and $50 \mu \mathrm{m}$ (striatum). b Spontaneous firing of dopaminergic neurons recorded in cell-attached mode in DAT$\mathrm{Cre}^{+/-}::$Arch3-GFP $+/-$mice with and without light (upper traces). Summary graph showing all experiments performed in DAT-Cre ${ }^{+/-:: A r c h 3-G F P}+/-$ mice with and without opto-inhibition. c 15 post-pre pairings induced tLTP $(n=8,6 / 8$ cells showed tLTP) in DAT-Cre $+/-:$ Arch3-GFP+/- mice without optostimulation (LED off), while opto-stimulation (LED on) during STDP pairings prevents tLTP induction $(n=6,1 / 6$ cells showed tLTP). This illustrates that eCB-tLTP requires DA release during STDP pairings. d 15 post-pre pairings induced tLTP with concomitant opto-stimulation (16 s duration) during STDP protocol in DAT-Cre $-/-:$ Arch3 $3^{+/+}$mice $(n=7,6 / 7$ cells showed tLTP). In panels $(\mathbf{b}-\mathbf{d})$, the yellow areas illustrate when LED was on to promote an optoinhibition. e, $\mathbf{f} 10$ post-pre pairings $\left(-30<\Delta t_{\text {STDP }}<0 \mathrm{~ms}\right.$ ) induced tLTP is $\mathrm{D}_{2} \mathrm{R}$-activation dependent. Summary of STDP experiments showing that eCBtLTP induced with 10 post-pre pairings $(n=10)$ is prevented by the co-application of antagonists of $D_{1} R$ and $D_{2} R, S C H 23390(4 \mu M)$ and sulpiride $(10 \mu M)$ $\left(n=9,8 / 9\right.$ cells showed tLTD and $1 / 9$ showed no plasticity) (e), and with the $\mathrm{D}_{2} \mathrm{R}$ antagonist, sulpiride $(10 \mu \mathrm{M}, n=8,0 / 8$ cells showed tLTP and $5 / 8$ showed tLTD) but tLTP was left unaffected by the sole application of the $D_{1} R$ antagonist, SCH23390 ( $4 \mu M, n=9,8 / 9$ cells showed tLTP) (f). Representative traces are the average of 15 EPSCs during baseline (black traces) and 45 min after STDP protocol (grey traces). Vertical grey dashed line indicates the STDP protocol. Error bars represent sem. ${ }^{\star} p<0.05$; ${ }^{\star \star} p<0.01 ;{ }^{\star \star \star} p<0.001$; ns: not significant by one sample $t$-test (c-f) 
membrane potential $\left(-19 \pm 5 \mathrm{mV}, n=5\right.$; with $\left.I_{\text {inj }}=100 \mathrm{pA}\right)$. We first verified that few pairings successfully induced tLTP (without photostimulation) in DAT-Cre ${ }^{+/-}:$Arch3-GFP $P^{+/-}$mice; we observed tLTP with 15 post-pre pairings $(143 \pm 12 \%, p=0.0091$, $n=8$; Fig. 3 c). $5-10$ post-pre pairings (with a single postsynaptic spike) were sufficient to induce potent tLTP in rat whereas in C57BL/6 mice 15 pairings (with $2-3$ postsynaptic spikes) were necessary to trigger tLTP. In DAT-Cre ${ }^{+/-}:: A r c h 3-G F P^{+/-}$mice, photostimulation concomitantly with the STDP protocol prevented tLTP $(102 \pm 8 \%, p=0.7943, n=6$; Fig. $3 c)$. Here, the $16 \mathrm{~s}$ photostimulation of Arch3 was far below artifactual local transmitter release reported for long $(5 \mathrm{~min})$ Arch3 activation ${ }^{41}$. As a control we ensured that photostimulation itself in DAT-Cre ${ }^{-/-}:$: Arch3-GFP ${ }^{+/-}$mice did not impair tLTP induction: tLTP was induced with 15 post-pre pairings applied concomitantly with photostimulation (140 $\pm 12 \%, p=0.0151, n=7$; Fig. $3 \mathrm{~d}$ ). Therefore, dopamine release during the STDP pairings is necessary for the induction of eCB-tLTP.

eCB-tLTP and -tLTD depend on distinct dopamine receptor. In the striatum, the principal subtypes of dopamine receptors are $D_{1}$ and $D_{2}$ receptors ${ }^{33}$. These receptors have opposite effects: $D_{1} R$ activates adenylyl cyclase and PKA via $G_{\text {olf }}$-coupled receptor signaling, and $D_{2} R$ inhibits them via $G_{i / o}$-coupled receptor. We questioned which dopaminergic receptor subtype was involved in eCB-tLTP induced by 10 post-pre pairings. Bath-applied SCH23390 $\left(4 \mu \mathrm{M}\right.$, a $\mathrm{D}_{1} \mathrm{R}$ antagonist) and sulpiride $(10 \mu \mathrm{M}$, a $\mathrm{D}_{2} \mathrm{R}$ antagonist) prevented eCB-tLTP. Indeed, we even observed a depression instead of tLTP ( $77 \pm 4 \%, p=0.0002, n=9$; Fig. 3e). We next selectively inhibited either $D_{1} R$ or $D_{2} R$. When we inhibited $\mathrm{D}_{1} \mathrm{R}$ (SCH23390), we observed tLTP $(169 \pm 21 \%$, $p=$ $0.0113, n=9$ ) while no tLTP could be elicited with sulpiride (73 $\pm 8 \%, p=0.0117, n=8$ ) (Fig. 3f). Hence, we observed that the prolonged inhibition of $\mathrm{D}_{2} \mathrm{R}$ (as well as both $\mathrm{D}_{1} \mathrm{R}$ and $\mathrm{D}_{2} \mathrm{R}$ ) flipped tLTP into tLTD. In an attempt to determine whether $\mathrm{D}_{2} \mathrm{R}$ and $\mathrm{CB}_{1} \mathrm{R}$ share a common, parallel, or cooperative pathway ${ }^{42}$, we next bath-applied quinpirole $(10 \mu \mathrm{M})$, a $\mathrm{D}_{2} \mathrm{R}$ agonist, concomitantly with 10 post-pre pairings, and observed tLTP (146 \pm $16 \%, p=0.0334, n=6$; Supplementary Fig. 3$)$, which was not different from the control tLTP $(p=0.1871)$. This observation is not in favor of cooperative or common pathways hypothesis for $\mathrm{D}_{2} \mathrm{R}$ and $\mathrm{CB}_{1} \mathrm{R}$, but points more likely toward parallel pathways ${ }^{42}$.

Secondly, we compared the dependence on dopamine receptor subtypes of eCB-tLTP with eCB-tLTD. We observed that the eCB-tLTD induced by 100 pre-post pairings was impaired by SCH23390 and sulpiride, (106 $\pm 14 \%, p=0.6714, n=6$; Supplementary Fig. 4a). We next selectively inhibited either $D_{1} R$ or $D_{2} R$ subtypes. The plasticity induced by 100 pre-post pairings was lost $(105 \pm 16 \%, p=0.7405, n=8)$ when we inhibited $D_{1} R$ with SCH23390, whereas we observed tLTP, instead of tLTD, with sulpiride ( $199 \pm 36 \%, p=0.0335, n=7$; Supplementary Fig. $4 \mathrm{~b})$.

Altogether, these experiments indicate that eCB-tLTP is $\mathrm{D}_{2} \mathrm{R}$ mediated but does not depend on $\mathrm{D}_{1} \mathrm{R}$ whereas eCB-tLTD is mediated by both $\mathrm{D}_{1} \mathrm{R}$ and $\mathrm{D}_{2} \mathrm{R}$.

What is the location of the $D_{2} R$ required for eCB-tLTP? We next questioned the location of the $\mathrm{D}_{2} \mathrm{R}$ involved in the induction of eCB-tLTP. $\mathrm{D}_{2} \mathrm{R}$ are expressed at different locations in the dorsal striatum: postsynaptically in $\mathrm{D}_{2} \mathrm{R}$-expressing $\mathrm{MSNs}^{43}$ and presynaptically in cholinergic interneurons ${ }^{44}$, nigrostriatal dopaminergic neurons ${ }^{45}$, and glutamatergic cortical afferents ${ }^{46,47}$ (Fig. 4a). To identify the $\mathrm{D}_{2} \mathrm{R}$ involved in the eCB-tLTP, we opted for two complementary strategies: (1) we genetically-ablated selectively $\mathrm{D}_{2} \mathrm{R}$-MSN or cholinergic interneurons (using Cremediated expression of the diphtheria toxin receptor (DTR) and stereotaxic diphtheria toxin (DT) injection ${ }^{48}$ ) and 6-ODHAablated selectively dopaminergic cells in the medial forebrain bundle (MFB), and (2) we selectively knocked-out the $\mathrm{D}_{2} \mathrm{R}$ expressed at $\mathrm{D}_{2} \mathrm{R}-\mathrm{MSN}$, cholinergic interneurons, dopaminergic cells, or corticostriatal glutamatergic terminals, and next examined whether eCB-tLTP could still be observed (Fig. 4a). Note that it was not possible to use the first strategy (i.e., genetic ablation) for corticostriatal afferents without impairing the corticostriatal transmission.

$D_{2} R$ on $D_{2} R$-MSNs are not required for eCB-tLTP. We first questioned the postsynaptic localization of the $\mathrm{D}_{2} \mathrm{R}$ involved in eCB-tLTP at the level of the MSNs (Fig. 4a). Due to the segregation of expression of $D_{1} R$ and $D_{2} R$ among MSNs in mice $\left(D_{1} R\right.$-like and $D_{2} R$-like for the direct and indirect pathways, respectively) ${ }^{43}$, roughly half of the $\mathrm{MSNs}$ are expected to be $\mathrm{D}_{2} \mathrm{R}$-expressing neurons. If eCB-tLTP was supported by the postsynaptic $\mathrm{D}_{2} \mathrm{R}$ MSNs, one would expect to induce eCB-tLTP in $\sim 50 \%$ of the (randomly chosen) recorded MSNs. eCB-tLTP was successfully induced in $83 \%(n=27)$ of the (randomly chosen) recorded MSNs, which does not favor the hypothesis of involvement of $\mathrm{D}_{2} \mathrm{R}$ expressed by MSNs in eCB-tLTP. To confirm this, we used transgenic $D_{1} R$-eGFP mice to induce eCB-tLTP specifically in $\mathrm{D}_{1} \mathrm{R}-\mathrm{eGFP}{ }^{+}$or non- $\mathrm{D}_{1} \mathrm{R}-\mathrm{eGFP}{ }^{+} \mathrm{MSNs}$ (Fig. $4 \mathrm{~b}, \mathrm{c}$ ). We observed tLTP in both $\mathrm{D}_{1} \mathrm{R}$-eGFP ${ }^{+}(148 \pm 8 \%, p=0.0033$, $n=6$; Fig. $4 \mathrm{~b})$ and non- $\mathrm{D}_{1} \mathrm{R}-\mathrm{eGFP}{ }^{+}(150 \pm 7 \%, p=0.0006, n=7$; Fig. 4c) MSNs, indicating that tLTP can be induced in both striatopallidal and striatonigral MSNs and that a postsynaptic $\mathrm{D}_{2} \mathrm{R}$ in MSNs is a priori less likely to be involved in eCB-tLTP.

To further test the involvement of the $\mathrm{D}_{2} \mathrm{R}-\mathrm{MSNs}$, we selectively ablated these neurons by Cre-mediated expression of DTR and DT injection ${ }^{48}$. To inactivate $\mathrm{D}_{2} \mathrm{R}$ in the specific neuronal population in the striatum of adult mice we use genetic ablation to avoid developmental effect or compensation. Moreover, it has been shown that the ablation of $40-45 \%$ of the striatal neurons in adult mice has no noticeable effect on others neuronal populations ${ }^{48}$. We placed Cre recombinase under the control of $\mathrm{A}_{2 \mathrm{~A}} \mathrm{R}$ promoter because in the striatum $\mathrm{A}_{2 \mathrm{~A}} \mathrm{R}$ expression is restricted to $\mathrm{D}_{2} \mathrm{R}-\mathrm{MSN}$. The DT was stereotaxically injected into the dorsal striatum to produce ablation of $\mathrm{D}_{2} \mathrm{R}-\mathrm{MSN}$. In those $\mathrm{A}_{2 \mathrm{~A}^{-}} \mathrm{Cre} \mathrm{C}^{+/-}:: \mathrm{iDTR} \mathrm{R}^{+/-}:: \mathrm{Drdla}-\mathrm{GFP}^{+/-}$transgenic mice after stereotaxic injection of DT, we found no detectable $\mathrm{D}_{2} \mathrm{R}^{+}$ cells but only $\mathrm{D}_{1} \mathrm{R}-\mathrm{GFP}$ MSNs, whereas in the contra-lateral hemisphere where DT had not been injected both populations of $\mathrm{D}_{1} \mathrm{R}^{+}$- and $\mathrm{D}_{2} \mathrm{R}^{+}-\mathrm{MSN}$ s were observed (Fig. $4 \mathrm{~d}$ ). Whole-cell recordings of $\mathrm{D}_{1} \mathrm{R}-\mathrm{GFP}$ MSNs, from $A_{2 A^{-}} \mathrm{Cr}^{+l-}:: i D T R^{+/-}::$ Drd1a-GFP ${ }^{+/-}$mice injected bilaterally with DT, showed that 15 post-pre pairings were still able to cause tLTP (146 \pm $7 \%, p=0.0007, n=7$; Fig. $4 \mathrm{e})$. As a control, we similarly injected DT in mice lacking DTR expression $\left(A_{2 A^{-}} \mathrm{Cr}^{-/-}:: i D T R^{+1-}::\right.$ Drdla-GFP $\left.{ }^{+/-}\right)$and also observed tLTP $(137 \pm 10 \%, p=0.0174$, $n=6$; Supplementary Fig. 5 a) that was not different from tLTP obtained with ablation of $\mathrm{D}_{2} \mathrm{R}-\mathrm{MSN}(p=0.3566)$.

We next generated selective conditional knock-out (cKO) mice

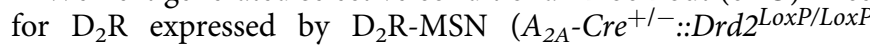
mice). We observed expression of tLTP induced by 15 postpre pairings in MSNs from $A_{2 A^{-}} C r e^{+/-}:: D r d 2^{L o x P / L o x P}$ mice ( $181 \pm 24 \%, p=0.0192, n=6$; Fig. $4 \mathrm{f})$. As the Cre driver control, we used the $A_{2 A}-C r e^{+l-}:: D r d 2^{W t / W t}$ mice and observed tLTP following 15 post-pre pairings ( $172 \pm 18 \%, p=0.0070, n=7$; Fig. 4g), which was not different from tLTP observed in $A_{2 A}-C r e^{+l-}:: D r d 2^{\text {LoxP } / L o x P}$ mice $(p>0.9999)$.

These results show that postsynaptic $\mathrm{D}_{2} \mathrm{R}$ located on $\mathrm{D}_{2} \mathrm{R}$ MSNs are not required for eCB-tLTP but thus should rely on the activation of presynaptically located $\mathrm{D}_{2} \mathrm{R}$. 
a
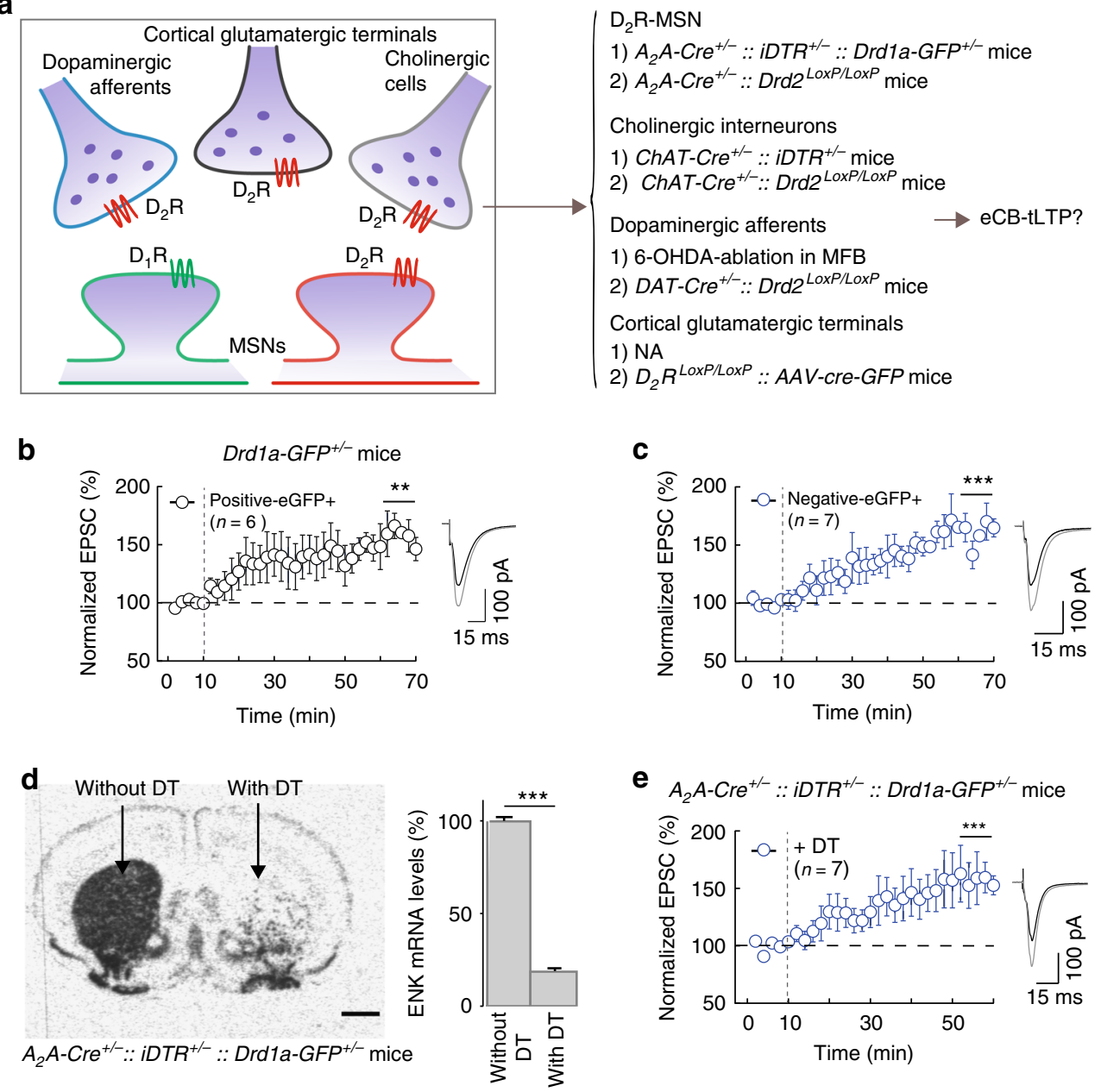

e $A_{2} A-C r e^{+/}:: i D T R^{+/-}::$Drd1a-GFP $P^{+/}$mice
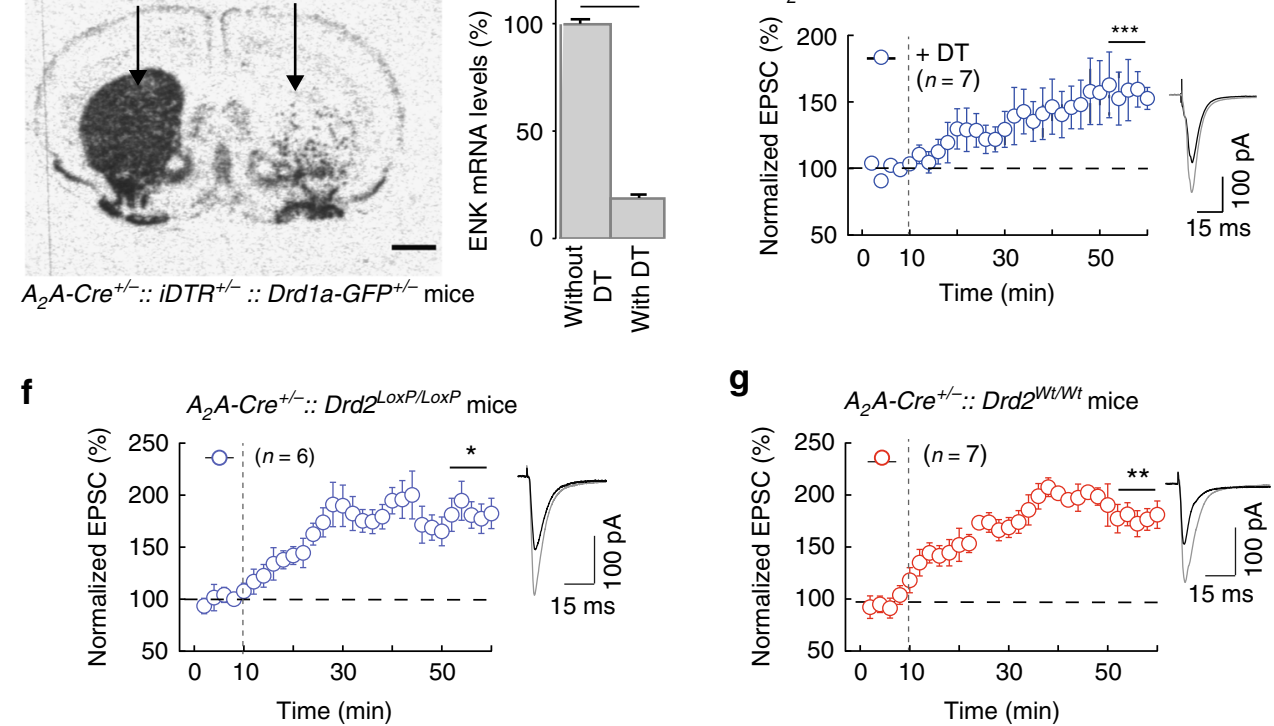

Fig. 4 eCB-tLTP does not depend on $D_{2} R$ expressed by $D_{2} R-M S N s$. a Location of $D_{2} R$ in striatum expressed in striato-pallidal MSNs, cholinergic interneurons, SNc dopaminergic and cortical glutamatergic afferents. Right: the two complementary strategies to identify the $D_{2} R$ involved in eCB-tLTP: (1) genetic-ablation of $D_{2} R-M S N$ or cholinergic interneurons (using DTR and stereotaxic DT injection) and 6-ODHA-ablation of dopaminergic cells in the $M F B$, and (2) $D_{2} R-c K O$ in $D_{2} R-M S N$, cholinergic interneurons, dopaminergic cells, or corticostriatal glutamatergic afferents; NA accounts for the impossibility to use a genetic-ablation of corticostriatal afferents without losing the studied EPSCs. $\mathbf{b}, \mathbf{c}$ tLTP induced with 15 post-pre pairings is observed in both striato-nigral ( $D_{1} R$-eGFP positive neurons, $D_{1} R$-eGFP ${ }^{+}, n=6,5 / 6$ cells showed tLTP) (b) and striato-pallidal ( $D_{1} R$-eGFP negative neurons, non$D_{1} R_{-}$eGFP ${ }^{+}, n=7,7 / 7$ cells showed tLTP) (c) MSNs. d, e Experiments of plasticity expression in genetically-ablated mice for $A_{2} A$-expressing neurons in the dorsal striatum. $\mathbf{d}$ In situ hybridization autoradiograms (left) and quantification (right bar graph, $n=5$ mice) of enkephalin mRNA in striatum caudal level of coronal sections in $A_{2 A^{-}}-C r e^{+/-}:: i D T R^{+/-}:: D r d 7 a-G F P^{+/-}$mice injected with DT stereotaxically in the dorsal striatum (right hemisphere) and without DT injection (left hemisphere). Scale bars: $1000 \mu \mathrm{m}$. e In mice in which $D_{2} R-M S N s$ were ablated $\left(A_{2 A}-C r e^{+/-:: i D T R^{+/}-:: D r d 7 a-G F P+/-}\right.$ mice injected with DT stereotaxically in the dorsal striatum) tLTP was induced with 15 post-pre pairings ( $n=7,7 / 7$ cells showed tLTP). $\mathbf{f}, \mathbf{g}$ Experiments of plasticity expression in selective $c K O$ for $D_{2} R$ expressed by striatal $D_{2} R-M S N s$. $\mathbf{f}$ In mice in which $D_{2} R$ was specifically knocked-out in $D_{2} R-M S N s\left(A_{2 A}-C r e+/-:\right.$. $\operatorname{Drd}{ }^{\text {LoxP } / \text { LoxP }}$ mice) tLTP was induced with 15 post-pre pairings $(n=6,5 / 6$ cells showed tLTP). $\mathbf{g}$ tLTP was observed in mice serving as the Cre driver control ( $A_{2 A}-C r e^{+/-}: . D r d 2 W t / W t$ mice, $n=7,6 / 7$ cells showed tLTP). Representative traces are the average of 15 EPSCs during baseline (black traces) and $45 \mathrm{~min}$ after STDP protocol (grey traces). Vertical grey dashed line indicates the STDP protocol. Error bars represent sem. ${ }^{\star} p<0.05 ;{ }^{\star \star} p<0.01$; ${ }^{\star \star \star} p<0.001$ by one sample $t$-test $(\mathbf{b}, \mathbf{c}-\mathbf{g})$ or Wilcoxon Signed rank test $(\mathbf{b})$ 
$D_{2} R$ on cholinergic interneurons are not required for eCBtLTP. Next, we first selectively ablated striatal cholinergic interneurons by Cre-mediated expression of DTR and DT injection to test the participation of the $\mathrm{D}_{2} \mathrm{R}$ express by striatal cholinergic interneurons in eCB-tLTP (Fig. 5a). Cre recombinase was under the control of ChAT promoter because in the striatum ChAT expression is restricted to cholinergic interneurons. Ablation of cholinergic interneurons was induced by DT, which was stereotaxically injected into the dorsal striatum. We verified that in ChAT-Cre ${ }^{+l-}:: i D T R^{+/-}$mice, there was no detectable ChAT cells after stereotaxic injection of DT whereas in the contra-lateral hemisphere (without DT injection) a positive ChAT staining was observed (Fig. 5a). In ChAT-Cre $e^{+/-}:: i D T R^{+/-}$mice injected bilaterally with DT, 15 post-pre pairings induced tLTP $(161 \pm$ $20 \%, p=0.0182, n=8$; Fig. $5 \mathrm{~b}$ ). We verified that this tLTP was $\mathrm{D}_{2}$ R-mediated by applying sulpiride $(10 \mu \mathrm{M})$ and no plasticity was observed ( $99 \pm 10 \%, p=0.9160, n=6$; Fig. $5 b)$. As a further control, we similarly injected DT in mice lacking DTR expression $\left(\right.$ ChAT-Cre $\left.e^{-/-}:: i D T R^{+l-}\right)$ and observed a tLTP $(150 \pm 13 \%, p=$ $0.0171, n=5$; Supplementary Fig. $5 \mathrm{~b}$ ) that was similar to tLTP obtained with ablation of cholinergic interneurons $(p=0.8221)$.

In the second step, we generated selective cKO mice for $\mathrm{D}_{2} \mathrm{R}$ expressed in cholinergic interneurons (ChAT-Cre ${ }^{+1-}:$ Drd $2^{\text {LoxP/ }}$ LoxP mice). In ChAT-Cre ${ }^{+-}:: D r d 2^{\text {LoxP/LoxP }}$ mice, 15 post-pre pairings induced tLTP in MSNs (169 $\pm 18 \%, p=0.0111, n=6$; Fig. 5c). As the Cre driver control, we used the ChAT-Cre ${ }^{+/-}:$: $\operatorname{Drd} 2^{W t / W t}$ mice and observed tLTP following 15 post-pre pairings $(135 \pm 9 \%, p=0.0175, n=5$; Fig. $5 d$ ), which was not different from tLTP observed in ChAT-Cre ${ }^{+/-}:$Drd2 $2^{\text {LoxP } / L o x P}$ mice $(p=0.0823)$.
These results indicate that eCB-tLTP does not depend on $\mathrm{D}_{2} \mathrm{R}$ activation located in cholinergic interneurons.

$D_{2} R$ on dopaminergic neurons are not required for eCB-tLTP. To evaluate the potential role of presynaptic $\mathrm{D}_{2} \mathrm{R}$ in the dopaminergic neurons, we first lesioned dopaminergic neurons with 6OHDA. We performed in vivo stereotaxic 6-OHDA injection (with desipramine) within the MFB of $\mathrm{P}_{35}$ rats (Fig. 6a) and striatal TH staining was decreased by $77 \pm 3 \%(p<0.0001, n=8)$ 2 weeks after lesion at $\mathrm{P}_{50}$ (Fig. 6b). In parallel, we used control $\left(\mathrm{P}_{50}\right.$ rats without any surgery) and sham-operated $\left(\mathrm{P}_{35}\right.$ rats with saline injection instead of 6-OHDA and recorded at $\mathrm{P}_{50}$ ) animals (Fig. $6 c$ and Supplementary Fig. 5c). We first verified that tLTP was induced with 10 post-pre pairings in $\mathrm{P}_{50}$ control rats (158 \pm $16 \%, p=0.0168, n=6$; Supplementary Fig. $5 \mathrm{c})$ and shamoperated rats $(147 \pm 10 \%, p=0.0032, n=7$; Fig. $6 \mathrm{c})$; both tLTP show similar magnitude $(p=0.7133)$. As expected, in 6-OHDA animals we did not observe any plasticity with 10 post-pre pairings ( $90 \pm 7 \%, p=0.1916, n=7$; Fig. $6 c$ ). We next bath-applied quinpirole $(10 \mu \mathrm{M})$, a selective agonist of $D_{2} R$, to overcome the lack of dopamine, and tLTP was observed (141 $\pm 9 \%, p=0.0076$, $n=6$; Fig. $6 \mathrm{~d}$ ); this tLTP was not different from those observed in sham-operated rats $(p=0.7984)$. In conclusion, tLTP could be observed (with quinpirole) in 6-OHDA-lesioned animals.

In the second step, we generated selective cKO mice for $\mathrm{D}_{2} \mathrm{R}$ expressed in dopaminergic cells (DAT-Cre ${ }^{+/-}:$Drd2 $2^{\text {LoxP/LoxP }}$ mice). Whole-cell recordings of MSNs from $D A T-C r e^{+/-}:$: $\operatorname{Drd} 2^{\text {LoxP }} /$ LoxP mice showed that 15 post-pre pairings induced tLTP $(154 \pm 15 \%, p=0.0166, n=6$; Fig. $6 \mathrm{e})$. As the Cre driver a
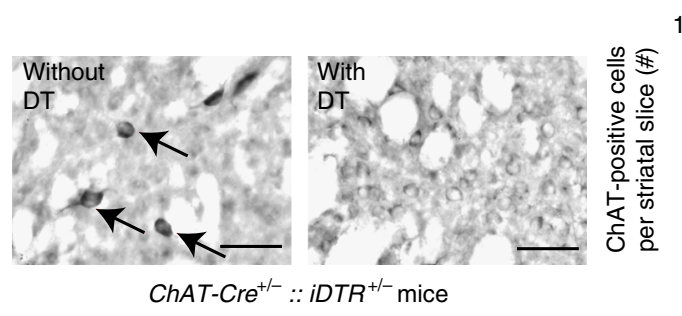

C

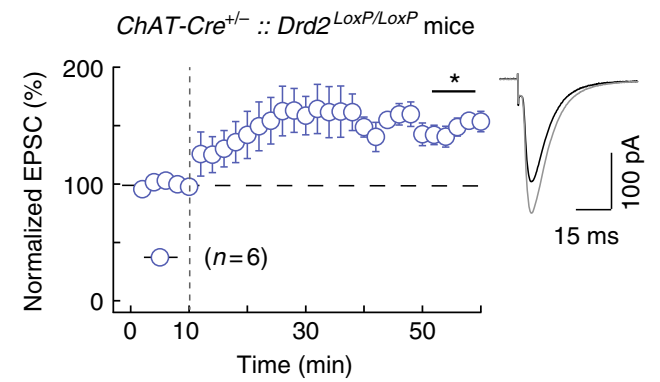

b

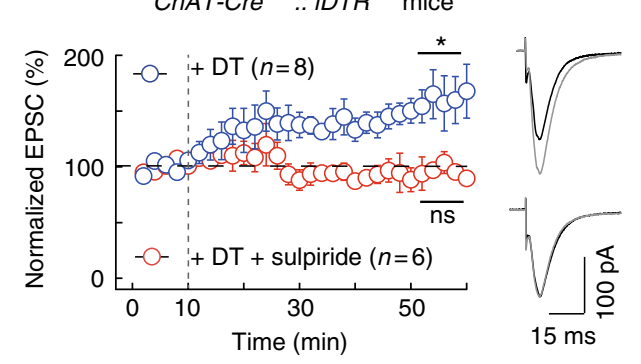

d

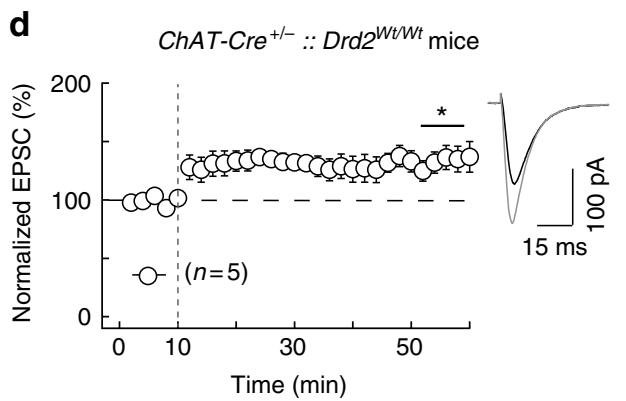

Fig. 5 eCB-tLTP does not depend on $D_{2} R$ expressed by cholinergic interneurons. $\mathbf{a}$, $\mathbf{b}$ Experiments of plasticity expression in genetically-ablated mice for

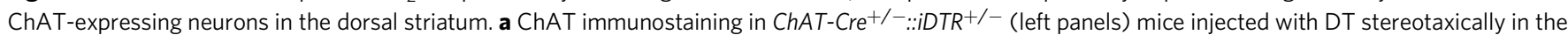
dorsal striatum (right hemisphere) and without DT injection (left hemisphere); quantification (right bar graph) of the number of Ach-positive cells per striatal slices $\left(n=6\right.$ mice). b tLTP was induced with 15 post-pre pairings in ChAT-Cre $+/-:: i D T R^{+/-}$mice $(n=8,8 / 8$ cells showed tLTP) injected bilaterally with diphtheria toxin in the dorsal striatum. LLTP observed in cholinergic interneuron-ablated mice was $D_{2} R$-mediated because it was prevented by sulpiride $\left(10 \mu \mathrm{M}, n=6,2 / 6\right.$ cells showed tLTP). c, $\mathbf{d}$ STDP expression in selective cKO for $D_{2} R$ expressed by cholinergic interneurons. $\mathbf{c}$ In mice in which $D_{2} R$ was

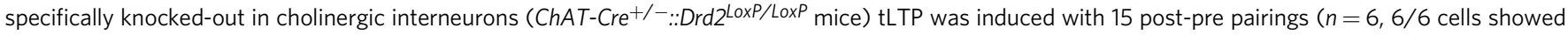
tLTP). $\mathbf{d}$ tLTP was observed in mice serving as the Cre driver control (ChAT-Cre ${ }^{+/-}:$:Drd $2^{W t / W t}$ mice, $n=5 ; 4 / 5$ cells showed tLTP). Representative traces are the average of 15 EPSCs during baseline (black traces) and $45 \mathrm{~min}$ after STDP protocol (grey traces). Vertical grey dashed line indicates the STDP protocol. Error bars represent sem. ${ }^{\star} p<0.05$; ${ }^{\star \star \star} p<0.001$; ns: not significant by Wilcoxon Signed rank test (a) or one sample $t$-test (b-d) 
a

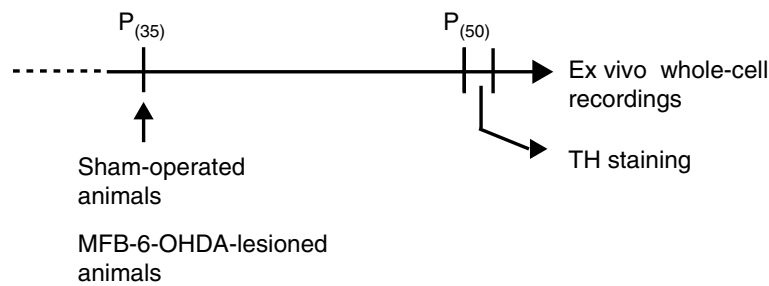

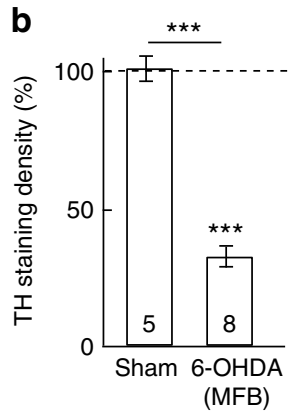

C

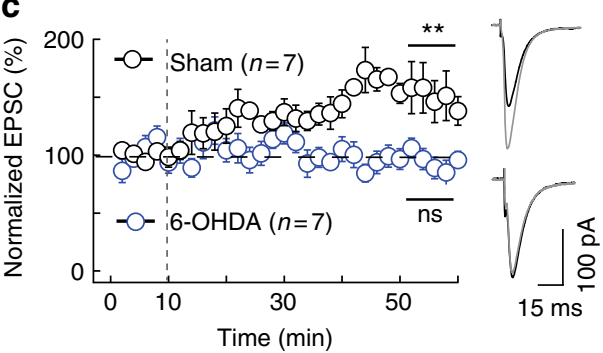

e

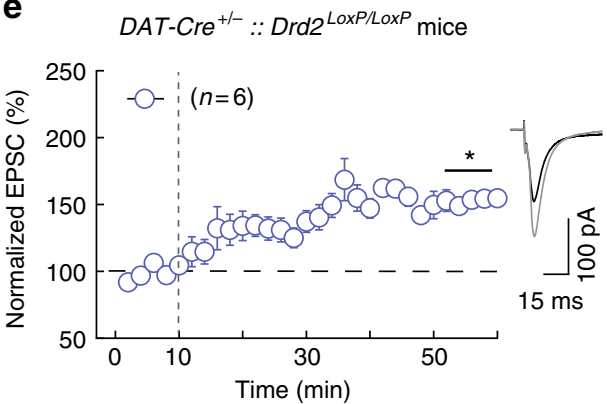

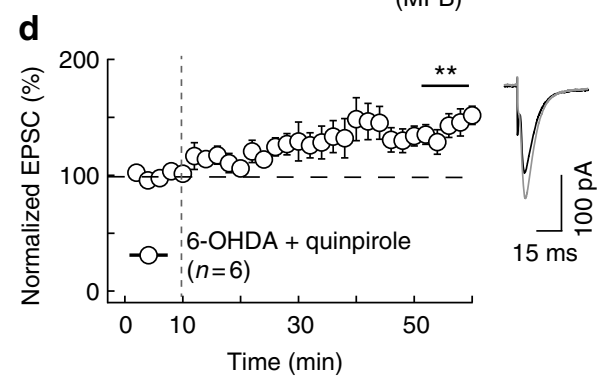

f $\quad D A T-C r e^{+-}:: D r d 2^{W t W t}$ mice

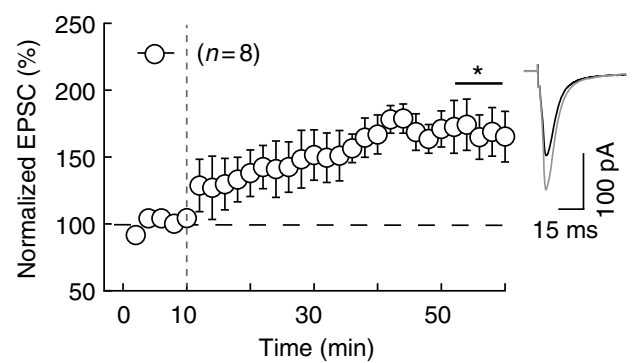

Fig. 6 eCB-tLTP does not depend on $D_{2} R$ expressed by dopaminergic neurons. a-d Experiments of plasticity expression in 6-ODHA-ablated dopaminergic cells in the MFB. a Scheme of the protocol showing the time course of the in vivo 6-OHDA lesions and ex vivo electrophysiological recordings. $\mathbf{b}$ Unilateral 6-OHDA injection in MFB led to degeneration of dopaminergic nigral neurons, illustrated by a loss of their striatal afferences as illustrating by the summary bar graph of TH immunostaining quantification. Sham-operated rats display equivalent TH staining in both striata. c 10 post-pre pairings induced tLTP in sham-operated ( $n=7,7 / 7$ cells showed tLTP) rats whereas tLTP was impaired in 6-OHDA-lesioned rats $(n=7,1 / 7$ cells showed tLTP), and $\mathbf{d}$ rescued with a $D_{2} R$ agonist, quinpirole $(10 \mu M, n=6,5 / 6$ cells showed tLTP). e, $\mathbf{f}$ STDP expression in selective cKO for D2R expressed by dopaminergic neurons. e In mice in which $D_{2} R$ was specifically knocked-out in dopaminergic neurons (DAT-Cre ${ }^{+/-}:$Drd2 ${ }^{\text {LoxP } / \text { LoxP }}$ mice) tLTP was induced with 15 post-pre pairings $\left(n=6,6 / 6\right.$ cells showed tLTP). $\mathbf{f}$ tLTP was observed in mice serving as the Cre driver control (DAT-Cre ${ }^{+}-: . D r d 2^{W t} / W t$ mice, $n=8 ; 6 / 8$ cells showed tLTP). Representative traces are the average of 15 EPSCs during baseline (black traces) and 45 min after STDP protocol (grey traces). Vertical grey dashed line indicates the STDP protocol. Error bars represent sem. ${ }^{\star} p<0.05$; ${ }^{\star \star} p<0.01$; ${ }^{\star \star \star} p<0.001$; ns: not significant by Wilcoxon Signed rank test (b) or one sample $t$-test (c-f)

control, we used the DAT-Cre ${ }^{+/-}:: D r d 2^{W t / W t}$ mice and observed tLTP following 15 post-pre pairings $(169 \pm 22 \%, p=0.0157, n=$ 8; Fig. 6f), which was not different from tLTP observed in DAT$\mathrm{Cr} \mathrm{C}^{+/-}:$Drd $2^{\text {LoxP } / \text { LoxP }}$ mice $(p=0.7193)$.

Altogether, these results show that presynaptic $\mathrm{D}_{2} \mathrm{R}$ located on nigrostriatal dopaminergic afferents are not required for eCB-tLTP.

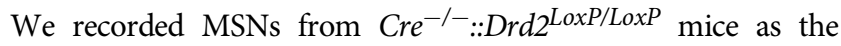
floxed gene control for the selective $\mathrm{D}_{2} \mathrm{R}-\mathrm{cKO}$ mice experiments. 15 post-pre pairings induced tLTP in $\mathrm{Cr} e^{-/-}:$Drd2 $2^{\text {LoxP/LoxP }}$ mice $(156 \pm 12 \%, p=0.0052, n=7$; Supplementary Fig. $5 d)$. We did not observe any difference between tLTP recorded in MSNs in the floxed gene control $\left(C r e^{-/-}:: D r d 2^{\operatorname{LoxP} / L o x P}\right.$ mice) and the Cre driver controls $\left(A_{2 A^{-}} C r e^{+/-}:: D r d 2^{W t / W t}, C h A T-C r e^{+l-}:: D r d 2^{W t / W t}\right.$, and DAT-Cre ${ }^{+l-}:: D r d 2^{W t / W t}$ mice) (ANOVA, $\left.p=0.5524\right)$.

eCB-tLTP depends on $D_{2} R$ in corticostriatal pyramidal cells. We next selectively inactivated $\mathrm{D}_{2} \mathrm{R}$ at corticostriatal glutamatergic afferents by cKO to test the implication of the $\mathrm{D}_{2} \mathrm{R}$ expressed by neocortical pyramidal cells in eCB-tLTP (Fig. 7). Bilateral $\mathrm{KO}$ of $\mathrm{D}_{2} \mathrm{R}$ in pyramidal cells was obtained by the stereotaxic injection of AAV-cre-GFP (AAV1.CMV.HI.eGFP-Cre. $W P R E . S V 40)$ in the layer 5 of the somatosensory cortex of $D_{2} R^{\text {LoxP/LoxP }}$ mice (Fig. 7a). In $D_{2} R^{\text {LoxP/LoxP }}$ mice injected with $A A V$-cre-GFP, we did not observe detectable positive immunostaining for $\mathrm{D}_{2} \mathrm{R}$ expression in pyramidal cells (Fig. $7 \mathrm{~b}$ ), whereas positive cells were observed in $D_{2} R^{\text {LoxP/LoxP }}$ mice injected with $A A V$-GFP (Fig. 7c); these later mice serving as control. Wholecell recordings of MSNs from $D_{2} R^{\text {LoxP/LoxP }}$ mice injected bilaterally with $A A V$-cre-GFP, showed that 15 post-pre pairings failed to induce tLTP, but tLTD was observed instead $(60 \pm 7 \%$, $p=0.0008, n=8$; Fig. $7 d$ ). As a control, we similarly injected $A A V$-GFP in $D_{2} R^{\text {LoxP/LoxP }}$ mice and observed tLTP induced by 15 post-pre pairings $(150 \pm 8 \%, p=0.0025, n=5)$ (Fig. 7e). These results demonstrate that eCB-tLTP depends on presynaptic $\mathrm{D}_{2} \mathrm{R}$-activation in cortical terminals. 

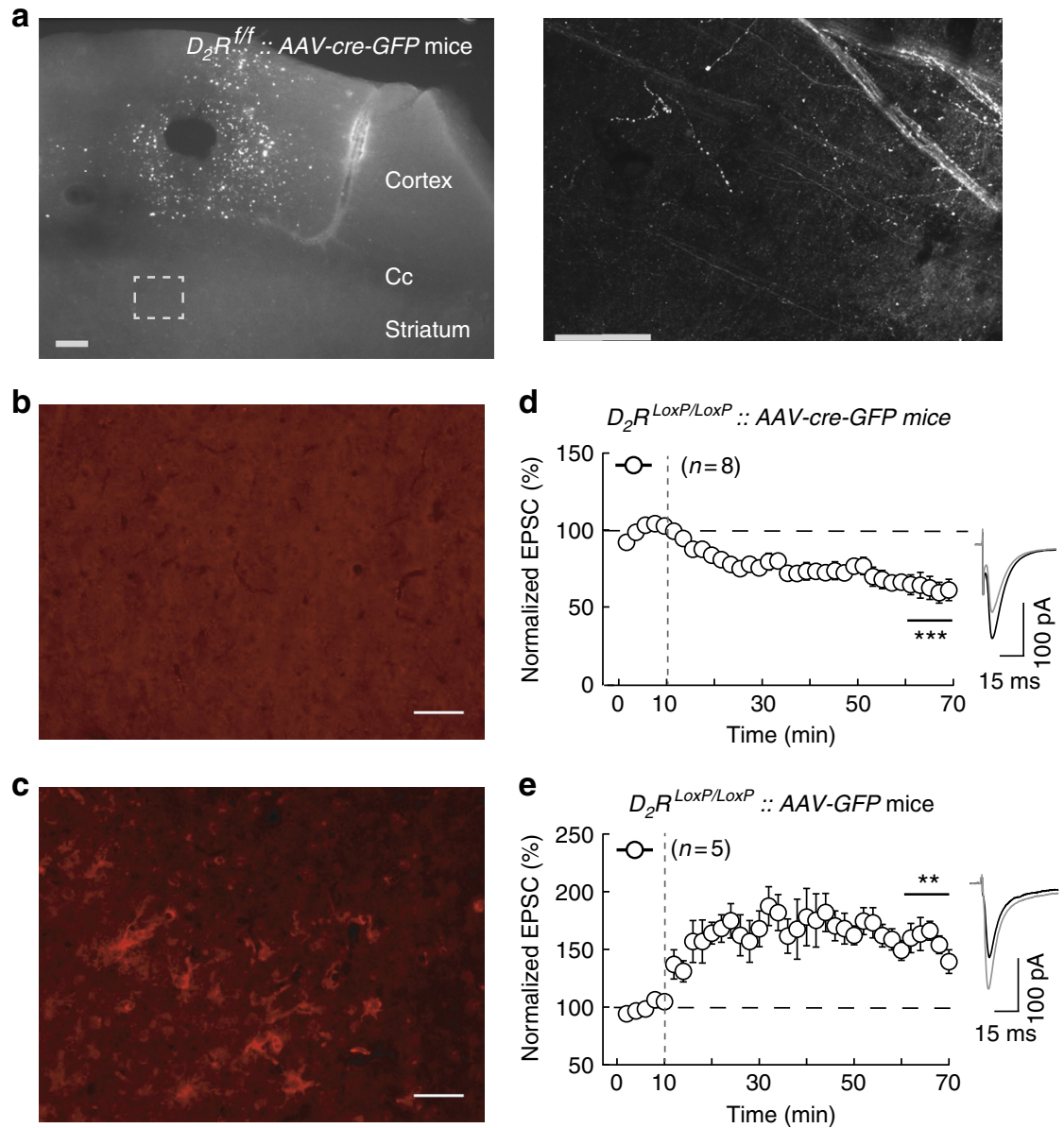

Fig. 7 eCB-tLTP depends on $D_{2} R$ expressed by cortical pyramidal cells. a GFP fluorescence in $D_{2} R^{\text {LoxP/LoxP }}$ injected with AAV-cre-GFP in somatosensory cortex. Left panel: site of injection of the AAV-cre-GFP and the resulting fluorescence in pyramidal cells in somatosensory cortex (scale bar: $250 \mu \mathrm{m}$ ). Note that the fluorescence is restricted to neurons within cortex and does not cross corpus callosum (Cc). Right panel: GFP fluorescence in corticostriatal afferents within dorsal striatum (scale bar: $100 \mu \mathrm{m}$ ). b, c $D_{2} R$ immunostaining in the somatosensory cortex of $D_{2} R^{\text {LoxP/LoxP }}$ mice injected with $A A V$-creGFP (b) or with AAV-GFP (c); (scale bar: $100 \mu \mathrm{m}$ ). d, e Experiments of plasticity expression in cKO mice for $\mathrm{D}_{2} \mathrm{R}$ expressed at corticostriatal glutamatergic afferents. $\mathbf{d} 15$ post-pre pairings failed to induce tLTP in $\mathrm{D}_{2} \mathrm{R}^{\mathrm{LoxP} / \text { LoxP }}$ mice injected with AAV-cre-GFP in somatosensory cortex $(n=8,0 / 8$ and $7 / 8$ cells showed tLTP and tLTD, respectively). e As a control, tLTP was induced with 15 post-pre pairings in $D_{2} R^{\text {LoxP/LoxP }}$ mice injected with AAV-GFP ( $n=5,5 / 5$ cells showed tLTP). Representative traces are the average of 15 EPSCs during baseline (black traces) and 45 min after STDP protocol (grey traces). Vertical grey dashed line indicates the STDP protocol. Error bars represent sem. ${ }^{\star \star} p<0.01 ;{ }^{\star \star \star} p<0.001$ by one sample $t$-test $(\mathbf{d}, \mathbf{e})$

Dopamine and eCB-tLTP interaction: a mathematical model. To provide hypotheses for the effects of the $\mathrm{D}_{2} \mathrm{R}$ inhibition/ deletion/cKO (Figs. 4-7), we developed a biologically plausible mathematical model ${ }^{13}$ of corticostriatal synaptic plasticity. Our model emulates the temporal dynamics of the signaling pathways involved in corticostriatal STDP $12,13,28,29,31,32$, combining a pathway leading from NMDAR to calmodulin and CaMKII with a second one that links postsynaptically mGluR5 and cytosolic calcium to $\mathrm{eCB}$ production and results in the activation of presynaptic $\mathrm{CB}_{1} \mathrm{R}$ (Fig. 8a). Our model expresses chemical kinetics for the reactions in these two pathways (Fig. 8a). Repeated pairing stimulations of the pre- and postsynaptic neurons trigger transient changes of the species implicated in the biochemical reaction network illustrated (Fig. 8a) according to standard massaction law kinetics. Hence, the temporal evolution of each species emerges from the coupling between the reaction network and the stimulations. We used the amount of phosphorylated postsynaptic CaMKII as a proxy for the postsynaptic contribution to the synaptic weight, while presynaptic $\mathrm{G}_{\mathrm{i} / \mathrm{o}}$-GPCR activation by $\mathrm{CB}_{1} \mathrm{R}$ and $\mathrm{D}_{2} \mathrm{R}$ was taken as a proxy for the presynaptic contribution (see Methods). The total synaptic weight $\left(W_{\text {total }}\right)$ was computed as the product of pre- and postsynaptic contributions ${ }^{13}$. Our model accounts for the outcome of plasticity when the spike timing $\left(\Delta t_{\mathrm{STDP}}\right)$, the frequency $\left(F_{\text {pairings }}\right)$, and the number $\left(N_{\text {pairings }}\right)$ of pairings are varied. The $W_{\text {total }}$ changes for $F_{\text {pairings }}=1 \mathrm{~Hz}$ and a range of $-35<\Delta t_{\mathrm{STDP}}<+35 \mathrm{~ms}$ and for 1 $<N_{\text {pairings }}<100$ values are illustrated by the color-map of Fig. $8 \mathrm{~b}$. In agreement with experimental data ${ }^{12,13}$, the outcome of plasticity is splitted along three plasticity domains: a first tLTP (i.e., eCB-tLTP) domain for $-3<\Delta t_{\text {STDP }}<-25 \mathrm{~ms}$ and $3<N_{\text {pairings }}<$ 30 , a second tLTP (i.e., NMDAR-tLTP) domain for $-10<\Delta t_{\text {STDP }}$ $<-25 \mathrm{~ms}$ and $N_{\text {pairings }}>50$, and a tLTD (i.e., eCB-tLTD) domain for $10<\Delta t_{\mathrm{STDP}}<25 \mathrm{~ms}$ and $N_{\text {pairings }}>20$. The quality of the match between model prediction and electrophysiology measurements is illustrated in Fig. 8c, d (see ref. ${ }^{13}$, for a more thorough account).

We next examined the dependence of plasticity on the activation of dopamine receptors, since our model integrates the dependence of plasticity on presynaptic $\mathrm{D}_{2} \mathrm{R}$ (see Methods). We compared the changes of eCB-tLTP domain under presynaptic $\mathrm{D}_{2} \mathrm{R}$ inhibition in the model against the data. Figure $8 \mathrm{e}$ illustrates the changes experienced by the two eCB-controlled domains when the level of presynaptic $\mathrm{D}_{2} \mathrm{R}$ activation decreases progressively from 100 to $0 \%$ of its control value $\left(\gamma_{\mathrm{D} 2 \mathrm{R}}=0.84\right.$, 
a

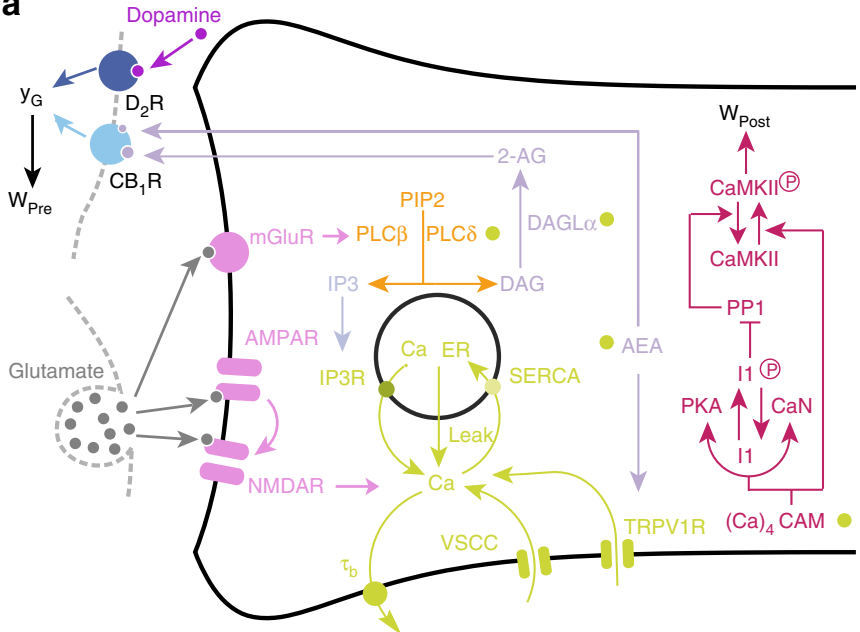

b

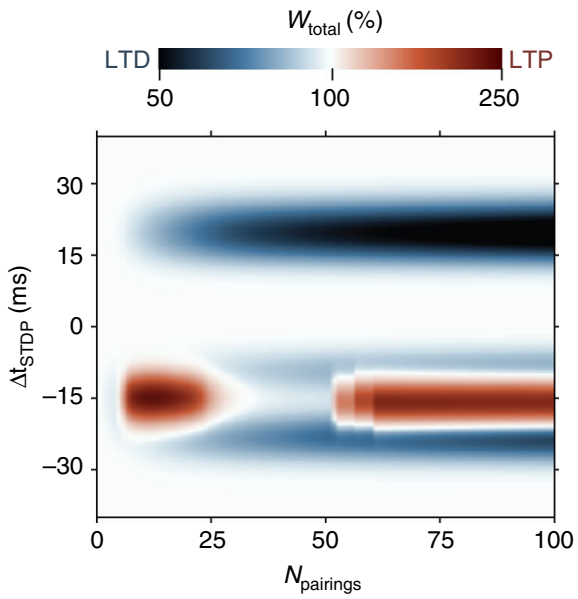

C

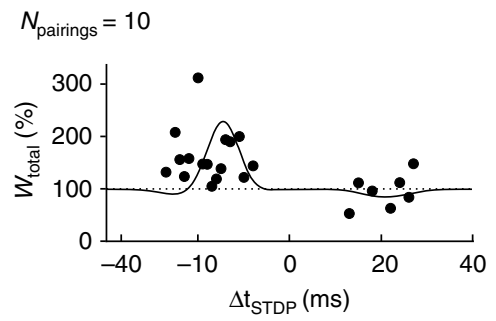

$\mathbf{d}_{N_{\text {pairings }}}=100$

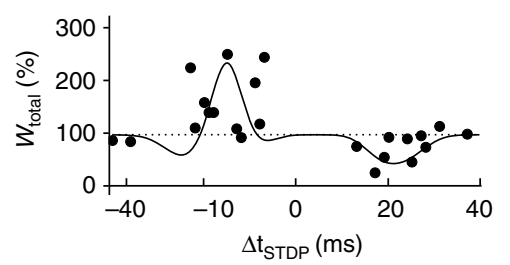

- Model

- STDP

experiments

e

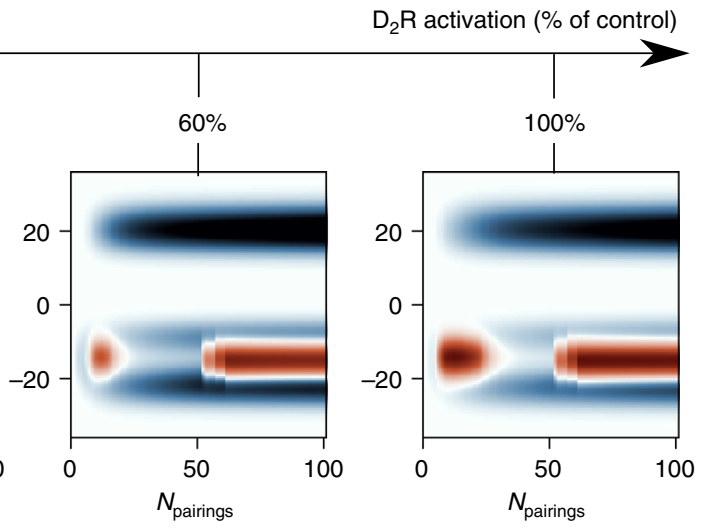

f

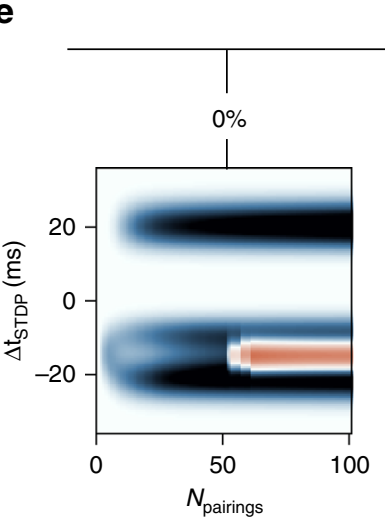

$\mathrm{D}_{2} \mathrm{R}$ activation (\% of control):

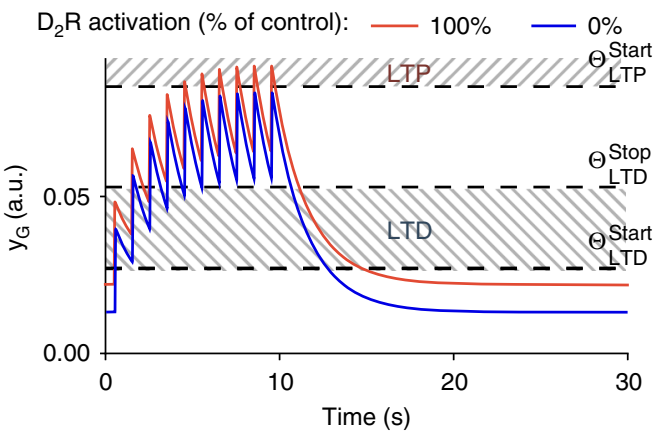

g

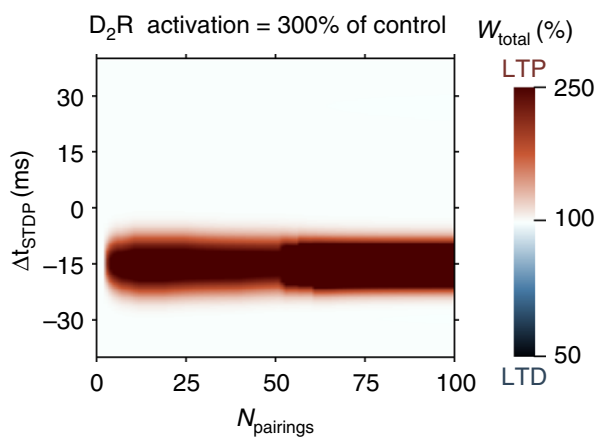

0.50, 0.25, and 0.00; see Methods). The eCB-tLTD domain (for $\Delta t_{\text {STDP }}$ around $+20 \mathrm{~ms}$ ) is not affected by the decrease of presynaptic $\mathrm{D}_{2} \mathrm{R}$ signaling. In contrast, eCB-tLTP (centered on $N_{\text {pairings }}=15$ and $\Delta t_{\mathrm{STDP}}=-15 \mathrm{~ms}$ ) is altered by a reduction of presynaptic $\mathrm{D}_{2} \mathrm{R}$ signaling: eCB-tLTP magnitude first decreases and is then replaced by eCB-tLTD. Therefore, our model predicts that blockers of presynaptic $\mathrm{D}_{2} \mathrm{R}$ do not affect eCB-tLTD and convert eCB-tLTP into tLTD; the latter prediction matches with the experimental data (Figs. 3f and $7 d$ ).

We next analyzed the dynamics of the model to explain this progressive change of eCB-tLTP to no plasticity then to eCBtLTD as $\mathrm{D}_{2} \mathrm{R}$ inactivation increases. The variable $y_{G}$ summarizes the effects of $C_{1} R$ and $D_{2} R$ activation on the presynaptic weight $\left(W_{\text {pre }}\right)$. We assumed that the effects of $\mathrm{D}_{2} \mathrm{R}$ and $\mathrm{CB}_{1} \mathrm{R}$ are 
Fig. 8 Threshold-based model interaction between dopamine and eCB-STDP. a Scheme of the modeled signaling network. The NMDAR-based pathway sets the postsynaptic weight $W_{\text {post }}$ as the phosphorylation state of CaMKIl $\alpha$. In the second pathway, coincident activation of phospholipase-C $\beta$ by postsynaptic mGluR and calcium entry via VSCC and TRPV1 induces eCB production (2-AG and AEA). eCBs activates $C B_{1} R$ which modulates $W_{\text {pre }}$ and $W_{\text {total }}=W_{\text {post }} \times W_{\text {pre. }}$ In the model, $D_{2} R$ in the presynaptic cortical neurons co-localize with $C B_{1} R$, so the effects of $D_{2} R$ and $C B_{1} R$ activations cumulate to determine whether the threshold for eCB-tLTP is reached. As a result, $D_{2} R$ activation by dopamine also contributes to $W_{\text {pre }}$ changes. Green disks indicate calcium-dependent steps. For a thorough description, see ref. ${ }^{13}$. Abbreviations: PIP2 phosphatidylinositol 4,5-biphosphate; DAG diacylglycerol; IP3 inositol-1,4,5-triphosphate; PLC $\beta / \delta$ phospholipase- $C \beta / \delta$; DAGL $\alpha$ diacylglycerol lipase- $\alpha$; 2-AG 2-arachidonoylglycerol; AEA anandamide; IP3R IP3-receptor channel; SERCA sarcoplasmic/endoplasmic reticulum calcium ATPase; $\mathrm{Ca}_{\mathrm{ER}}$ calcium in the endoplasmic reticulum; (Ca) ${ }_{4} \mathrm{CaM}$ fully bound calmodulin; CaN calcineurin aka PP2B; I1p/l1 phosphorylated/unphosphorylated protein phosphatase-1 inhibitor-1 (DARPP-32 in MSNs); PP1 protein phosphatase-1; CaMKII $\mathrm{Ca}^{2+} /$ calmodulin-dependent protein kinase-II. b Model prediction for $W_{\text {total }}$ changes (LTP and LTD) with varying $N_{\text {pairings }}$ and $\Delta t_{\text {STDP }}$ (at $1 \mathrm{~Hz}$ ) shows eCB$\operatorname{tLTP}\left(3<N_{\text {pairings }}<40,-10<\Delta t_{\mathrm{STDP}}<-25 \mathrm{~ms}\right)$ and the eCB-tLTD domains $\left(N_{\text {pairings }}>70,+10<\Delta t_{\mathrm{STDP}}<+25 \mathrm{~ms}\right)$. c, d Changes of $W_{\text {total }}$ with $\Delta t_{\mathrm{STDP}}$, for $N_{\text {pairings }}=10(\mathbf{c})$ or $100(\mathbf{d})$ pairings at $1 \mathrm{~Hz}$. Black lines are simulation results whereas the black circles show experimental measurements. e $W_{\text {total }}$ change map of $\mathbf{b}$ when presynaptic $D_{2} R$ activation is reduced from 100 to $0 \%$ of the control (shown in $\mathbf{b}$ ). $\mathbf{f}$ Temporal evolution of $y_{\mathrm{G}}$, that combines the effects of $D_{2} R$ and $C B_{1} R$ activation during STDP protocol (10 pairings at $1 \mathrm{~Hz}, \Delta t_{S T D P}=-15 \mathrm{~ms}$ ). LTD is triggered when $y_{\mathrm{G}}$ is between $\theta_{\mathrm{LTD}}^{\text {start }}$ and $\theta_{\mathrm{LTD}}^{\text {stop }}$ whereas $\mathrm{LTP}$ is triggered when $y_{G}>\theta_{L T P}^{\text {start }} D_{2} R$ activation was $100 \%$ (red full line) or $0 \%$ (blue full line). g Model prediction for $W_{\text {total }}$ upon hyperdopaminergy, with a threefold increase of tonic dopamine (DA, see Supplementary Experimental Procedures) compared to the control case shown in $\mathbf{b}$. All other parameters were as in $\mathbf{b}$

cumulative, up to the tLTP threshold. We expressed this assumption mathematically by the linear combination of three terms: $y_{G}=k_{\mathrm{CB} 1 \mathrm{R}} \cdot x_{\mathrm{CB} 1 \mathrm{R}}+\gamma_{\mathrm{D} 2 \mathrm{R}} \cdot \mathrm{DA}+\gamma_{\text {other }}$. The first term, presynaptic $\mathrm{CB}_{1} \mathrm{R}$ activation $\left(x_{\mathrm{CB} 1 \mathrm{R}}\right)$ is controlled by postsynaptically-produced eCBs (see Methods), the dynamics of which comprises calcium-dependent biochemical reactions. Since postsynaptic calcium dynamics in our model emerges from the interaction between the paired stimulations and the biochemical network, $y_{G}$ dynamics indirectly depends on spike timing via the calcium-dependence of $x_{\mathrm{CB} 1 \mathrm{R}}$. The second term depends on $\mathrm{D}_{2} \mathrm{R}$ activation $\left(\gamma_{\mathrm{D} 2 \mathrm{R}} \cdot \mathrm{DA}\right)$ and, the third one, $\gamma_{\text {other }}$ accounts for background activation of the $\mathrm{G}_{\mathrm{i} / \mathrm{o}}$-GPCR pathway not related to $\mathrm{D}_{2} \mathrm{R}$ and $\mathrm{CB}_{1} \mathrm{R}$.

Here, $W_{\text {pre }}$ is set by $y_{G}$ relatively to three plasticity thresholds $\left(\theta_{\mathrm{LTD}}^{\text {start }}, \theta_{\mathrm{LTD}}^{\text {stop }}\right.$, and $\left.\theta_{\mathrm{LTP}}^{\text {start }}\right): W_{\text {pre }}$ drops (LTD) when $y_{G}$ is in between $\theta_{\mathrm{LTD}}^{\text {start }}$ and $\theta_{\mathrm{LTD}}^{\text {stop }}$, whereas $W_{\text {pre }}$ rises (LTP) if $y_{G}$ becomes larger than $\theta_{\text {LTP }}^{\text {start }}$ (Fig. $8 f$, dashed line). In control conditions (Fig. 8f, red line), the combination of the effect of $\mathrm{CB}_{1} \mathrm{R}$ activation by eCBs (produced upon the STDP protocol) and that of $\mathrm{D}_{2} \mathrm{R}$ by (tonic) dopamine, build up to yield $y_{G}$ levels (red) that overcome $\theta_{\text {LTP }}^{\text {start }}$ resulting in LTP. However, with $\mathrm{D}_{2} \mathrm{R}$ activation blocked (Fig. 8f, blue line), $y_{G}$ cannot reach $\theta_{\mathrm{LTP}}^{\text {start }}$, thus effectively preventing the expression of eCB-tLTP. Nevertheless, $y_{G}$ still crosses the LTD range with LTD accumulating in proportion to the time spent between $\theta_{\mathrm{LTD}}^{\text {start }}$ and $\theta_{\mathrm{LTD}}^{\text {stop }}$. As a result, eCB-tLTD is expressed instead of eCB-tLTP. This is in agreement with our experimental data (Figs. 3f and 7d) in which the prolonged inhibition or deletion of $\mathrm{D}_{2} \mathrm{R}$ switched eCB-tLTP into tLTD, whereas brief (16 s) opto-inhibition prevented eCB-tLTP without conversion into tLTD (Fig. 3c). Therefore, according to the model, the switch from eCB-tLTP to eCB-tLTD observed when presynaptic $D_{2} R$ are blocked is due to the reduction of the synergestic effect of $D_{2} R$ on the presynaptic terminal that needs to be present in addition to the activation of the presynaptic $\mathrm{G}_{\mathrm{i} / \mathrm{o}}$-GPCR pathway by $\mathrm{CB}_{1} \mathrm{R}$ to reach $\theta_{\mathrm{LTP}}^{\text {start }}$. Thus, the presynaptic cortical $\mathrm{D}_{2} \mathrm{R}$ not only allow the expression of eCB-tLTP, but even control the polarity (LTP vs LTD) of the plasticity induced by a low number of pairings.

The above results (experimental and theoretical) consider $1 \mathrm{~Hz}$ stimulations. In the neocortex, increasing $F_{\text {pairings }}$ with large numbers of pairings promotes tLTP at the expense of $\operatorname{tLTD}^{49}$. We next used the model to address the effects of $\mathrm{D}_{2} \mathrm{R}$ block on STDP when $F_{\text {pairings }}$ varies (Supplementary Fig. 6). Note that our model was calibrated based on experimental data harvested at $1 \mathrm{~Hz}$ and does not account for the complex frequency-dependence of glutamate signaling (glutamate release and uptake, AMPAR desensitization). For this reason, our previous investigations ${ }^{13}$ have shown that sensitivity to frequency changes is larger in the model than in the experiments, so that the effects of a small change of $F_{\text {pairings }}$ in the model $(1.00-1.05 \mathrm{~Hz})$ are similar to the effects of larger changes $(1-3 \mathrm{~Hz})$ in the experiments (see Discussion). In control conditions ( $100 \% \mathrm{D}_{2} \mathrm{R}$ activation) and small numbers of pairings $\left(N_{\text {pairings }}<25\right.$ and $\left.\Delta t_{\text {STDP }}<0\right)$, eCBtLTP is not expressed when $F_{\text {pairings }}<0.9 \mathrm{~Hz}$. However, eCB-tLTP is not much altered above the threshold (Supplementary Fig. 6b, $6 \mathrm{~d}$ and $6 \mathrm{f}$ ). Note that eCB-tLTD observed at negative $\Delta t_{\text {STDP }}$ (blue areas in Supplementary Fig. 6b) is in all case negligible in control conditions for $N_{\text {pairings }}<25$. When presynaptic $\mathrm{D}_{2} \mathrm{R}$ are blocked (Supplementary Fig. 6a) the model shows a similar picture, but the frequency threshold for eCB-tLTP is larger than $1 \mathrm{~Hz}$. This explains the disappearance of eCB-tLTP at $1 \mathrm{~Hz}$ when $\mathrm{D}_{2} \mathrm{R}$ are blocked (Supplementary Fig. 6a). Moreover the faint eCB-tLTD present in control conditions at $\Delta t_{\text {STDP }}<0$ is amplified in the absence of $\mathrm{D}_{2} \mathrm{R}$ activation (Supplementary Fig. 6a, blue areas for $\left.N_{\text {pairings }}<25, \Delta t_{\mathrm{STDP}}<0\right)$. This explains the emergence of eCB-tLTD at $1 \mathrm{~Hz}$ when $\mathrm{D}_{2} \mathrm{R}$ are blocked.

The impact of $F_{\text {pairings }}$ on STDP is quite different with larger pairings $\left(N_{\text {pairings }}>50\right)$. In control conditions $\left(100 \% \quad \mathrm{D}_{2} \mathrm{R}\right.$ activation), the expression domain of NMDAR-tLTP $\left(N_{\text {pairing }}>\right.$ $\left.50, \Delta t_{\text {STDP }}<0\right)$ enlarges drastically when $F_{\text {pairings }}$ increases (Supplementary Fig. 6b and 6d). NMDAR-tLTP even invades the quadrant of positive $\Delta t_{\text {STDP }}$ (Supplementary Fig. 6f). This strengthening of NMDAR-tLTP with increased $F_{\text {pairings }}$ matches published experimental reports ${ }^{49,50}$. In comparison, the expression of eCB-tLTD with such large pairings $\left(N_{\text {pairings }}>50\right.$, $\left.\Delta t_{\mathrm{STDP}}>0\right)$ is not much altered by large frequencies. Since presynaptic $\mathrm{D}_{2} \mathrm{R}$ in the model only affects eCB plasticity, blocking $\mathrm{D}_{2} \mathrm{R}$ receptors has no effect on NMDAR-tLTP directly (Supplementary Fig. $6 \mathrm{a}, 6 \mathrm{c}$ and $6 \mathrm{e}, N_{\text {pairings }}>50, \Delta t_{\text {STDP }}<0$ ), although it strengthens eCB-tLTD, thus partially canceling NMDRtLTP. To summarize, at $F_{\text {pairings }}>1 \mathrm{~Hz}$ our model predicts that presynaptic $\mathrm{D}_{2} \mathrm{R}$ signaling fine-tunes STDP polarity (LTP or LTD) via the control of eCB-plasticity.

Hyperdopaminergy in the striatum is of interest since observed in drug addiction ${ }^{51}$. We next used our mathematical model to explore the effects of hyperdopaminergy on STDP. Figure $8 \mathrm{~g}$ shows model prediction when the level of tonic dopamine is increased threefold with respect to control. eCB-tLTP induction is hardly altered by hyperdopaminergy. The main modification is that larger $N_{\text {pairings }}$ can induce eCB-tLTP, so eCB-tLTP domain fuses with the NMDAR-tLTP domain. In contrast, hyperdopaminergy has a drastic effect on eCB-tLTD: the whole eCB-tLTD domain disappears so pre-post pairings fail to induce any 
plasticity regardless $N_{\text {pairings }}$ or $\Delta t_{\text {STDP }}$ (Fig. $8 \mathrm{~g}$ ). With large $\mathrm{D}_{2} \mathrm{R}$ activation, $y_{G}$ remains located between $\theta_{\text {LTD }}^{\text {stop }}$ and $\theta_{\text {LTP }}^{\text {start }}$, which is too large to induce LTD but too weak to trigger LTP. Therefore, the prediction of our model is that hyperdopaminergy (via activation of presynaptic cortical $\mathrm{D}_{2} \mathrm{R}$ ), should prevent eCB-tLTD and considerably extend the domain of expression of eCB-tLTP.

\section{Discussion}

Corticostriatal long-term plasticity provides a fundamental mechanism for the function of the basal ganglia in procedural learning ${ }^{14-19,21,52}$. We uncovered the existence of striatal eCBtLTP that relies on of $\mathrm{CB}_{1} \mathrm{R}$ and TRPV 1 activation and is induced by a low number of pairings ${ }^{12,13}$. eCB-tLTP requires high levels of $\mathrm{CB}_{1} \mathrm{R}$ activation that can be reached with $10-15$ post-pre pairings. Indeed, for such $N_{\text {pairings }}$, eCB synthesis and release would contribute maximally to presynaptic $\mathrm{CB}_{1} \mathrm{R}$ activation (maximal cytosolic calcium influx from NMDAR, VSCC, TRPV1, and maximal calcium efflux from internal stores, combined with a minimal $\mathrm{CB}_{1} \mathrm{R}$ desensitization) ${ }^{13}$. According to our mathematical model, beyond 30 post-pre pairings, calcium efflux from the internal calcium stores decreases (because of a sub-optimal calcium refilling of the internal stores) while $\mathrm{CB}_{1} \mathrm{R}$ desensitization increases concomitantly. $\mathrm{CB}_{1} \mathrm{R}$ activation then crosses $\theta_{\mathrm{LTP}}^{\text {start }}$ (Fig. 8f), so that eCB-tLTP vanishes, and NMDAR-tLTP can be expressed for post-pre $N_{\text {pairings }}>50$ (Fig. $8 \mathrm{~b}$; see ref. ${ }^{13}$ ). Interestingly, GABAergic microcircuits are not involved in eCB-tLTP induction or magnitude at corticostriatal synapses, but control eCB-tLTP polarity ${ }^{12}$. eCBs have been mainly reported to depress synaptic weight through the activation of $\mathrm{CB}_{1} \mathrm{R}$ or TRPV $1^{1,2}$. This view is now challenged by studies reporting opposite effects (potentiation) in various brain structures using various signaling pathways $^{3-13}$. We describe here and in previous studies ${ }^{11-13,50} \mathrm{a}$ tLTP in mammals, wherein eCB signaling directly underlies both the induction and the long-term maintenance of synaptic weight increase.

Our mathematical model is sensitive to $F_{\text {pairings }}$ (Supplementary Fig. 6). The results predicted by the model for small frequency changes (from 1.0 to $1.2 \mathrm{~Hz}$ ) match well the results we obtained experimentally with larger frequency changes (from 1 to $3 \mathrm{~Hz}$ ). This distortion may be due to the numerous frequencydependent mechanisms (glutamate release or uptake, AMPAR desensitization) that buffer the effects of $F_{\text {pairings }}$ increase in the experiments. For instance, the amount of glutamate released upon presynaptic stimulation does not depend on the frequency of presynaptic stimulation in the model. Therefore, the impact of increased frequency on the amplitude of the calcium trace is larger than in the experiments where presynaptic shortterm plasticity decreases the amount of released glutamate when presynaptic frequency increases ${ }^{53}$, thus compensating for the effects of frequency increase on the calcium traces. Those frequency-dependent mechanisms could have been accounted for but would have been at the expense of a further complexification of the model.

Dopamine is a key actor of action selection and associative learning 22 and for the modulation of striatal projection $24,25,33$. Electrical, chemical, or transcranial stimulation in different cortical areas enhances dopamine release in the corresponding projecting striatal areas including the dorsal striatum ${ }^{38-40}$. Our results show that dopamine is a key element for eCB-tLTP expression through the activation of $\mathrm{D}_{2} \mathrm{R}$. Contrarily to eCB-LTD induced with high- or low-frequency stimulation protocol ${ }^{24,25}$ (i.e., a rate-coding paradigm), eCB-tLTP (induced here with a low number of STDP pairings, i.e., a time-coding paradigm) does not require postsynaptic $D_{2} R$ expressed by striatopallidal neurons. Indeed, the selective ablation of those $\mathrm{D}_{2} \mathrm{R}-\mathrm{MSN}$ or the selective
$\mathrm{D}_{2} \mathrm{R}$ cKO in $\mathrm{D}_{2} \mathrm{R}-\mathrm{MSN}$ failed to prevent eCB-tLTP. Moreover, we observed eCB-tLTP in $\mathrm{D}_{1} \mathrm{R}$-expressing MSNs as well as $\mathrm{D}_{1} \mathrm{R}$ non-expressing cells. This demonstrates that $\mathrm{D}_{2} \mathrm{R}$ involved in eCB-tLTP have a presynaptic location. The presynaptic $\mathrm{D}_{2} \mathrm{R}$ are expressed at three different locations: the nigrostriatal dopaminergic afferents ${ }^{45}$, the cholinergic interneurons ${ }^{44}$, and the corticostriatal glutamatergic afferents ${ }^{46,47}$. Presynaptic $D_{2} R$ in nigrostriatal dopaminergic afferents and cholinergic interneurons are not required for eCB-tLTP as demonstrated by ablation or selective $\mathrm{D}_{2} \mathrm{R}$ cKO experiments. Although eCB-tLTP is muscarinic $\mathrm{M}_{1} \mathrm{R}$-mediated ${ }^{13}$, eCB-tLTP was observed with ablated cholinergic interneurons suggesting that $M_{1} R$ should have a constitutive activity ${ }^{54}$. Moreover, the involvement of $\mathrm{D}_{2} \mathrm{R}$ expressed by cholinergic interneurons is unlikely since the activation of those $\mathrm{D}_{2} \mathrm{R}$ would decrease acetylcholine release, promoting LTD, and not LTP ${ }^{55}$. eCB-tLTP was absent in mice in cKO of $\mathrm{D}_{2} \mathrm{R}$ in the somatosensory cortex. Those results unambiguously demonstrate that $\mathrm{D}_{2} \mathrm{R}$ implicated in the control of eCBtLTP are located on the presynaptic pyramidal neurons, where the $\mathrm{CB}_{1} \mathrm{R}$ are also expressed. Interestingly, eCB-LTD (rate-coded) has been reported to be tightly controlled by $D_{2} R$ expressed by striatal cholinergic interneurons ${ }^{55,56}$ or by $\mathrm{D}_{2} \mathrm{R}-\mathrm{MSNs} \mathrm{s}^{57,58}$ (for review see ref. ${ }^{24}$ ), i.e., distinct pools of $\mathrm{D}_{2} \mathrm{R}$ are engaged in eCB-LTD and eCB-LTP. In the nucleus accumbens, activation of $\mathrm{CB}_{1} \mathrm{R}$ on cortical terminals limits dopamine release and governs reward-driven behavior ${ }^{59}$. Dopamine-eCB interactions remain to be examined in dorsal striatum in the temporal-credit assignment problem ${ }^{60-62}$.

Colocalization of $\mathrm{D}_{2} \mathrm{R}$ and $\mathrm{CB}_{1} \mathrm{R}$ on the same presynaptic terminals would act synergistically on adenylate cyclase activity and promote eCB-tLTP or eCB-tLTD induced by small numbers of pairings, depending on the activation of $\mathrm{D}_{2} \mathrm{R}$ and $\mathrm{CB}_{1} \mathrm{R}$. Our results suggest a joint $C_{1} R$ - and $D_{2} R$-control of STDP whereby the same signal, adenylate cyclase or PKA activity, can trigger tLTP or tLTD depending on the dynamics of the signaling pathway $^{13}$. A similar hypothesis has been proposed for NMDARdependent STDP where the outcome of plasticity depends on the dynamics of intracellular calcium ${ }^{63,64}$. However our results unravel further complexity of the system, since in our hands the eCB-tLTD induced with 100 pairings shows additional dependence on $\mathrm{D}_{1} \mathrm{R}$ (both $\mathrm{D}_{2} \mathrm{R}$ - and $\mathrm{D}_{1} \mathrm{R}$-mediated). This result matches some of the earlier results on corticostriatal STDP ${ }^{28}$; but see the STDP with 100 pairings at $0.1 \mathrm{~Hz}$ in which tLTD was $\mathrm{D}_{1} \mathrm{R}$ - but not $\mathrm{D}_{2} \mathrm{R}$-dependent ${ }^{29}$. We observed that in both cases, pharmacological blocking of $\mathrm{D}_{2} \mathrm{R}$ gave rise to a switch of plasticity: with 100 pre-post pairings, tLTP was obtained instead of eCB-tLTD, whereas with 10 post-pre pairings $\mathrm{D}_{2} \mathrm{R}$ inhibition resulted in tLTD instead of eCB-tLTP; note that the inhibition of $\mathrm{D}_{1} \mathrm{R}$ has no effect on eCB-tLTP and prevents eCB-tLTD. Interestingly, only prolonged inhibition of $\mathrm{D}_{2} \mathrm{R}$ (pharmacological and genetic deletion experiments) switched eCB-tLTP into tLTD whereas a brief (16 s) opto-inhibition of dopamine release during STDP pairings prevented eCB-tLTP without inducing tLTD. We hypothesize that opto-inhibition likely prevents phasic dopamine release during STDP pairings without affecting much background tonic dopamine levels whereas prolonged inhibition of $\mathrm{D}_{2} \mathrm{R}$ would affect preferentially the tonic component. $\mathrm{D}_{2} \mathrm{R}$ have been widely described to efficiently sense the tonic dopamine because of their high affinity for dopamine ${ }^{65}$, but $\mathrm{D}_{2} \mathrm{R}$ can also encode phasic dopamine signals ${ }^{66}$. This suggests that depending on the level of the tonic dopamine, $\mathrm{D}_{2} \mathrm{R}$ would favor either eCB-tLTP or eCB-tLTD (for a low number of pairings). Thus, presynaptic cortical $\mathrm{D}_{2} \mathrm{R}$ operate as gatekeepers for the expression and polarity of corticostriatal eCB-STDP (eCB-tLTP and eCB-tLTD) depending on the activity pattern. This could be linked to the role of dopamine in the temporal credit-assignment by operating a 
retroactive control of STDP polarity, as demonstrated in striatum $^{60-62}$ or in hippocampus ${ }^{67}$.

Although, eCB-tLTP can be induced with 15 post-pre pairings in $\mathrm{D}_{1} \mathrm{R}$ - and $\mathrm{D}_{2} \mathrm{R}$-MSNs, it does not imply that in physiological conditions eCB-tLTP would be equally triggered in these MSN subpopulations. Indeed, $\mathrm{D}_{2} \mathrm{R}-\mathrm{MSN}$ are more excitable than $\mathrm{D}_{1} \mathrm{R}$ $\mathrm{MSNs}^{68}$ and thus would not integrate similarly incoming cortical inputs, especially in the frame of a plasticity induced by a very low number of pairings, such as eCB-tLTP. This differential membrane excitability may be exacerbated by a higher excitability of $\mathrm{D}_{2} \mathrm{R}$-MSN dendrites, allowing back-propagating action potentials to invade more efficiently distal dendrites in $\mathrm{D}_{2} \mathrm{R}-\mathrm{MSN}^{69}$. This is of importance for STDP in which a back-propagating action potential is triggered for every pairings. Therefore, one can expect that eCB-tLTP would be more prone to occur in $\mathrm{D}_{2} \mathrm{R}$ - than in $\mathrm{D}_{1} \mathrm{R}-\mathrm{MSN}$. It thus remains to investigate whether $\mathrm{D}_{2} \mathrm{R}-\mathrm{MSN}$ would have a proeminent role in the fast learning via expression of eCB-tLTP. The use of noisy STDP pairings, to approach invivo-like conditions, revealed that plasticity robustness depends on the signaling pathways with eCB-tLTP and eCB-tLTD being much more robust than NMDAR-tLTP ${ }^{50}$. In vivo conditions for eCB-tLTP emergence, using naturalistic firing patterns recorded across learning tasks still need to be determined.

Alterations of the $\mathrm{eCB}$ system seem to contribute to actionlearning defects in Parkinson's disease ${ }^{24,34}$. So far, this alteration was thought to rely on disruption of the well-characterized eCBLTD, which is $\mathrm{D}_{2} \mathrm{R}$-dependent ${ }^{24,25}$. Here, we report that eCBtLTP is also $\mathrm{D}_{2} \mathrm{R}$-dependent, is disrupted in a rodent model of Parkinson's disease, and can be rescued with L-DOPA treatment. $\mathrm{D}_{2} \mathrm{R}$ are central in various psychiatric diseases ${ }^{70}$. According to our findings, the effect of dopamine on corticostriatal STDP would vary with the cellular location (MSN, cholinergic, dopaminergic, or neocortical neurons) of $\mathrm{D}_{2} \mathrm{R}$ and on the thalamus/ cortical activity regimes. It remains to analyze the effects of the disruption of eCB-tLTP in vivo to address the roles of eCB-tLTP in physiological and pathophysiological states. From our mathematical model, it is expected that the eCB-tLTP expression domain extends considerably under hyperdopaminergia. One could thus speculate that psychostimulant drugs such as cocaine or amphetamines (which trigger a hyperdopaminergia) would promote eCB-tLTP and possibly facilitate the early phase of learning. On the contrary, upon hypodopaminergia, it is expected that LTD will take over instead of eCB-tLTP and thus affect the fast learning. These predictions remain to be tested in the frame of learning and memorizing salient events from a low number of spikes ${ }^{2}$. Because it is induced by small numbers of pairings, eCB-tLTP may represent a central molecular substrate for the rapid learning of new arbitrary associative memories and behavioral rules ${ }^{20}$ characterizing the flexible behavior of mammals or during initial stages of slower habit learnings 14,52 , which may possibly be disrupted in Parkinson's disease ${ }^{24,34}$.

\footnotetext{
Methods

Animals. Sprague-Dawley rats (Charles River, L'Arbresle, France) and C57BL/6 mice (DAT-Cre, Archeorhodopsin3-GFP, Adora2a-Cre, inducible-DTR, Drd1aGFP, ChAT-cre and $D_{2} R^{\text {LoxP/LoxP }}, A_{2 A}-C r e^{+/-}:$Drd2 $2^{\text {LoxP/LoxP }}$, ChAT-Cre $e^{+/-}:$: $\operatorname{Drd} 2^{\text {LoxP/LoxP }}$, DAT-Cre ${ }^{+/-}:$Drd2 $2^{\text {LoxP/LoxP }}$ mice) were used for electrophysiology, in situ hybridization, and immunohistochemistry. Adora $2 a-C r e^{+/-}$transgenic mice expressing the Cre recombinase under the control of the striatopallidal specific adenosine A2A receptor (Adora2a) promoter $^{48}$. C57BL/6 Adora2a-Cre ${ }^{+l-}$ mice were crossed with C57BL/6 $i D T R^{+/+}$mice $^{71}$, and Drd1a-GFP ${ }^{+/+}$resulting in $50 \%$ triple-heterozygous Adora2a-Cre $e^{+/-}:: i D T R^{+l-}:: D_{1} R-G F P^{+/-}$and $50 \%$ Adora2a$C r e^{-/-}:: i D T R^{+l-}:: D_{1} R-G F P^{+l-}$ which were used as controls. C57BL/6 ChAT-Cre $+/-$ (JAX SN6410) mice were crossed with C57BL/6 iDTR $R^{+/+}$mice $^{71}$. Drd2 floxed mice carrying two targeted loxP sites flanking Drd2 exon $2^{72}$. The Cre-mediated expression of Arch3 was obtained using mice expressing Cre-recombinase under the control of the Slc6a3 promoter (DAT-Cre mice, JAX SN006660) and Rosa26 LSL Arch3-GFP (Jackson Laboratory). Mice were housed by groups of 3-5 mice, in
}

a $12 \mathrm{~h} \mathrm{light/dark} \mathrm{cycle,} \mathrm{with} \mathrm{food} \mathrm{and} \mathrm{water} \mathrm{available} \mathrm{ad} \mathrm{libitum.} \mathrm{All} \mathrm{experiments}$ were performed in accordance with local animal welfare committee (CIRB and ULB Ethical Committees) and EU guidelines (Directive 2010/63/EU). Every precaution was taken to minimize stress and the number of animals used in each series of experiments.

Patch-clamp recordings and analysis. Corticostriatal connections (between somatosensory cortex layer 5 and dorsal striatum) are preserved in a horizontal plane $\mathrm{e}^{30}$. Horizontal brain slices with thickness of 330 or $300 \mu \mathrm{m}$ were prepared, respectively, from rats $\left(\mathrm{P}_{(20-25)}\right.$ for most experiments, $\mathrm{P}_{50}$ for 6-OHDA lesion, and $\mathrm{P}_{(60-65)}$ for Parkinson's disease animal model) or mice $\left(\mathrm{P}_{(25-45)}\right)$ using a vibrating blade microtome (VT1200S, Leica Microsystems, Nussloch, Germany). In a subset of experiments, we performed coronal brain slices to record in cell-attached dopaminergic neurons from the SNc. Brains were sliced in a $95 \% \mathrm{CO}_{2} / 5 \%$ $\mathrm{O}_{2}$-bubbled, ice-cold cutting solution containing (in $\mathrm{mM}$ ) $125 \mathrm{NaCl}, 2.5 \mathrm{KCl}$, 25 glucose, $25 \mathrm{NaHCO}_{3}, 1.25 \mathrm{NaH}_{2} \mathrm{PO}_{4}, 2 \mathrm{CaCl}_{2}, 1 \mathrm{MgCl}_{2}, 1$ pyruvic acid, and transferred into the same solution at $34^{\circ} \mathrm{C}$ for $1 \mathrm{~h}$ and next moved to room temperature.

Patch-clamp recordings were performed in the dorsolateral striatum ${ }^{12,32,35}$. Borosilicate glass pipettes of 4-6 M $\Omega$ resistance contained for whole-cell recordings (in $\mathrm{mM}$ ): $105 \mathrm{~K}$-gluconate, $30 \mathrm{KCl}, 10 \mathrm{HEPES}, 10$ phosphocreatine, $4 \mathrm{ATP}-\mathrm{Mg}, 0.3$ GTP-Na, 0.3 EGTA (adjusted to $\mathrm{pH} 7.35$ with $\mathrm{KOH}$ ). The composition of the extracellular solution was (mM): $125 \mathrm{NaCl}, 2.5 \mathrm{KCl}, 25$ glucose, $25 \mathrm{NaHCO}_{3}, 1.25$ $\mathrm{NaH}_{2} \mathrm{PO}_{4}, 2 \mathrm{CaCl}_{2}, 1 \mathrm{MgCl}_{2}, 10 \mu \mathrm{M}$ pyruvic acid bubbled with $95 \% \mathrm{O}_{2}$ and $5 \%$ $\mathrm{CO}_{2}$. Signals were amplified using EPC10-2 amplifiers (HEKA Elektronik, Lambrecht, Germany). All recordings were performed at $34^{\circ} \mathrm{C}$ using a temperature control system (Bath-controller V, Luigs \& Neumann, Ratingen, Germany) and slices were continuously superfused at $2-3 \mathrm{ml} / \mathrm{min}$ with the extracellular solution. Slices were visualized on an Olympus BX51WI microscope (Olympus, Rungis, France) using a $4 \times / 0.13$ objective for the placement of the stimulating electrode and a $40 \times / 0.80$ water-immersion objective for localizing cells for whole-cell recordings. Current- and voltage-clamp recordings were filtered at $5 \mathrm{kHz}$ and sampled at $10 \mathrm{kHz}$ using the Patchmaster v2x32 program (HEKA Elektronik).

Off-line analysis was performed using Fitmaster (Heka Elektronik) and Igor-Pro 6.0.3 (WaveMetrics, Lake Oswego, OR, USA). Statistical analysis was performed using Prism 5.0 software (San Diego, CA, USA). In all cases " $n$ " refers to the number of repetitions of an experiment (each experiment being performed on different brain slices) from single slice. Experimenters were blind to most of the mice genotype during electrophysiological recordings and analysis. All results were expressed as mean \pm sem, and statistical significance was assessed using Mann-Whitney test or the one sample $t$ test when appropriate at the significance level $(p)$ indicated.

Spike-timing-dependent plasticity induction protocols. Electrical stimulation was performed with a bipolar electrode (Phymep, Paris, France) placed in the layer 5 of the somatosensory cortex ${ }^{12,30}$. Electrical stimulation was monophasic at constant current (ISO-Flex stimulator, AMPI, Jerusalem, Israel). Currents were adjusted to evoke 50-200 pA EPSCs. Repetitive control stimuli were applied at 0.1 Hz. STDP protocols consisted in pairings of pre- and postsynaptic stimulations (at $1 \mathrm{~Hz})$ separated by a temporal interval $\left(\Delta t_{\mathrm{STDP}}\right)$. Presynaptic stimulations corresponded to cortical stimulations and the postsynaptic stimulation of an action potential evoked by a depolarizing current step (30 ms duration) in MSNs. MSNs were maintained all along the STDP experiments at a constant holding membrane potential which corresponds to their initial resting membrane potential $(-81.6 \pm$ $0.4 \mathrm{mV}, n=61$ ). Thus, across experiment EPSCs were measured at the same membrane potential (voltage-clamp mode); STDP pairings (current-clamp mode) were conducted at this same holding membrane potential. Neurons were recorded for $10 \mathrm{~min}$ during baseline and for at least $50 \mathrm{~min}$ after STDP protocol; long-term synaptic efficacy changes were measured from $50 \mathrm{~min}$. 30 successive EPSCs (at $0.1 \mathrm{~Hz}$ ) were individually measured and averaged. Variation of input and access resistances, measured every $10 \mathrm{~s}$ all along the experiment, beyond $20 \%$ led to the rejection of the experiment.

STDP protocol consisting in 5-10 post-pre pairings (with a single postsynaptic spike) were sufficient to induce potent tLTP in rat while in C57BL/6 mice 15 pairings (with $2-3$ postsynaptic spikes) were necessary to trigger tLTP.

Chemicals. N-(piperidin-1-yl)-5-(4-iodophenyl)-1-(2,4-dichlorophenyl)-4-methyl1H-pyrazole-3-carboxamide (AM251, $3 \mu \mathrm{M}$, Tocris) and (S-)-5-Aminosulfonyl-N[(1-ethyl-2-pyrrolidinyl)methyl]-2-methoxybenzamide (sulpiride, $10 \mu \mathrm{M}$, Tocris) and (2E)-N-(2,3-Dihydro-1,4-benzodioxin-6-yl)-3-[4-(1,1-dimethylethyl)phenyl]2-propenamide (AMG9810, $1 \mu \mathrm{M}$ ) were dissolved in ethanol and then added in the external solution at a final concentration of ethanol of $0.01-0.1 \% . \mathrm{R}(+)-7-\mathrm{Chloro}-$ 8-hydroxy-3-methyl-1-phenyl-2,3,4,5-tetrahydro-1H-3-benzazepine hydrochloride (SCH-23390, $4 \mu \mathrm{M}$, Sigma), (4aR-trans)-4,4a,5,6,7,8,8a,9-Octahydro-5-propyl-1Hpyrazolo[3,4-g]quinoline hydrochloride (quinpirole, $10 \mu \mathrm{M}$, Tocris) and $+\mathrm{N}-(2,6$ Dimethylphenylcarbamoylmethyl)triethylammonium chloride (QX-314, $10 \mu \mathrm{M}$, Tocris) were dissolved in water and added in the external solution. None of the bath-applied drugs had a significant effect on basal EPSC amplitudes: AM251 $(3 \mu \mathrm{M}): 101 \pm 5 \%(p=0.8610, n=7)$, co-application of SCH23390 $(4 \mu \mathrm{M})$ and 
sulpiride $(10 \mu \mathrm{M}): 102 \pm 3 \%,(p=0.5056, n=9)$ and quinpirole $(10 \mu \mathrm{M}): 98 \pm 2 \%$, $(p=0.3449, n=8)$.

Opto-inhibition of dopaminergic neurons. We bred C57BL/6 DAT-Cre $e^{+/-}$and C57BL/6 Arch3-GFP ${ }^{+/+}$mice (Jackson Laboratory) leading to mice that selectively expressed Archeo-rhodopsin in DAT-expressing neurons (DAT-Cre ${ }^{+1-}:$ Arch3$G F P^{+1-}$ mice); DAT-Cre $e^{-/-}:$Arch3-GFP $P^{+1-}$ mice were used as a control. $585 \mathrm{~nm}$ yellow-green light was delivered via field illumination using a high-power LED source ( $\mathrm{pE}$ excitation System, CoolLED, Andover, UK).

Double immunostaining for GFP (for Arch3 expression in dopaminergic neurons) and $\mathrm{TH}$. Brain slices $(300 \mu \mathrm{m})$ were fixed overnight in $2 \%$ paraformaldehyde at $4{ }^{\circ} \mathrm{C}$. Non-specific binding was blocked by incubating the slices for $2 \mathrm{~h}$ at room temperature in $10 \%$ normal goat serum (Merk-Millipore, Molsheim, France) in 2\% BSA and 1\% Triton X-100 solution. Brain slices were incubated with a pair of primary antibodies, chicken anti-GFP (1:2000, AB13970, Abcam, Paris, France) and mouse anti-TH (1:500, MAB318, Merk-Millipore), in $0.5 \%$ Triton $\mathrm{X}-100,1 \%$ BSA and $2.5 \%$ normal goat serum (overnight $4{ }^{\circ} \mathrm{C}$ ), and then with the secondary antibodies, goat anti-chicken Alexa (1:1000, A11039, Life Technology-Invitrogen, Villebon-sur-Yvette, France) and goat anti-mouse IgG1 (1:200, 1070-03, Southern Biotech-Clinisciences, Montrouge, France) (overnight $4{ }^{\circ} \mathrm{C}$ ).

Selective ablation of $\mathbf{D}_{\mathbf{2}} \mathbf{R}$-expressing MSNs. We bred C57BL/6 Adora2a-Cre $\mathrm{Al}^{+/-}$ and inducible $\mathrm{C} 57 \mathrm{BL} / 6 \mathrm{DTR}^{+/+}\left(\mathrm{iDTR}^{+/+}\right.$) mice leading to double heterozygous: mice that selectively expressed the DTR in $\mathrm{D}_{2} \mathrm{R}-\mathrm{MSN}$ (Adora2a-Cre ${ }^{+/-}:: i D T R^{+/-}$, $A_{2} A-D T R^{+l-}$ mice $)$ and Adora2a-Cre $e^{-/-}:: i D T R^{+l-}\left(A_{2} A-D T R^{-/-}\right.$mice $)$used as the corresponding control for electrophysiology ${ }^{48}$. A2A receptor promoter was chosen because specifically expressed in striatum only by $\mathrm{D}_{2} \mathrm{R}-\mathrm{MSNs}^{48}$. Inducible ablation allows a high spatial resolution (achieved with stereotaxic injections of toxin) and prevents developmental adaptations. We next bred Adora2a-Cre ${ }^{+/-}:$: $i D T R^{+/-}$mice with Drdla-GFP mice ${ }^{48}$ to ensure recording $\mathrm{D}_{1} \mathrm{R}-\mathrm{MSN}$. Adora2a-Cre ${ }^{+/-}:: i D T R^{+l-}:: D r d 1 a-G F P^{+l-}$ mice (and their control Adora2a$\left.C r e^{-/-}:: i D T R^{+/-}:: D r d 1 a-G F P^{+/-}\right)$. At the age of 4 weeks, $1 \mu$ of DT (SigmaAldrich) by side (diluted in PBS $0.01 \mathrm{M}$ to a concentration of $100 \mathrm{pg} / \mu \mathrm{l}$ ) was slowly stereotaxically injected at $0.25 \mu \mathrm{l} / \mathrm{min}$ with a blunt needle in four sides of the dorsal striatum with the following coordinates (with bregma as references): anterior +1.2 $\mathrm{mm}$, lateral $\pm 1.5 \mathrm{~mm}$, ventral $+3.6 \mathrm{~mm}$; or anterior $+0.5 \mathrm{~mm}$, lateral $\pm 1.8 \mathrm{~mm}$, ventral $+3.6 \mathrm{~mm}$. We reported an almost complete loss of striatal $\mathrm{A}_{2} \mathrm{~A}$ receptor binding from 14 days after DT injections ${ }^{48}$. Therefore, electrophysiological recordings were performed from 15-20 days after DT injections.

In situ hybridization for enkephalin $m R N A$ : Striatal $20 \mu \mathrm{m}$ coronal sections were cut from fresh-frozen brain (Adora2a-Cre ${ }^{+/-}:: i D T R^{+/-}:: D r d 1 a-G F P^{+/-}$mice) and mounted on glass slides. After the hybridization for enkephalin mRNA procedure $^{48}$, sections were exposed to Kodak Biomax MR film for 1-2 week(s) depending on the marker studied and digitalized images were generated from the autoradiograms. For quantification of in situ hybridization and binding (ImageJ software), an averaged optical density (OD) in different areas of interest was measured and background level was subtracted to obtain corrected values. Two to three sections were used for each animal to calculate the mean OD.

Selective ablation of ChAT interneurons. We bred C57BL/6 ChAT-Cre ${ }^{+/-}$and inducible C57BL/6 DTR $\mathrm{DT}^{+/+}\left(\mathrm{iDTR}^{+/+}\right)$mice leading to double heterozygous: mice that selectively expressed the DTR in cholinergic interneurons (ChAT-Cre ${ }^{+1-}::$

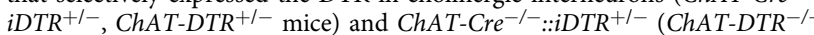
mice) used as the corresponding control for patch-clamp experiments. The ablation is performed in the same condition than $\mathrm{D}_{2} \mathrm{R}-\mathrm{MSNs}$. $1 \mu \mathrm{l}$ of DT (Sigma) by side (diluted in PBS $0.01 \mathrm{M}$ to a concentration of $100 \mathrm{pg} / \mu \mathrm{l}$ ) was stereotaxically injected at $0.25 \mu \mathrm{l} / \mathrm{min}$ with a blunt needle in four sides of the dorsal striatum with the following coordinates: anterior $+1.2 \mathrm{~mm}$, lateral $\pm 1.5 \mathrm{~mm}$, ventral $+3.6 \mathrm{~mm}$; or anterior $+0.5 \mathrm{~mm}$, lateral $\pm 1.8 \mathrm{~mm}$, ventral $+3.6 \mathrm{~mm}$. An almost complete loss of ChAT immunostaining in the striatum from 14 days after DT injections was observed and recordings were performed from 15-20 days after DT injections.

ChAT immunostaining: ChAT-Cre ${ }^{+1-}:: i D T R^{+/-}$mice were anesthetized and perfused transcardially with $0.01 \mathrm{M}$ PBS, followed by $4 \%$ paraformaldehyde for tissue fixation. Brains were dissected, dehydrated in $20-30 \%$ sucrose solutions and frozen. Coronal free-floating sections of $30 \mu \mathrm{m}$ thickness were cut through the striatum with a freezing microtome (Leica). Sections were treated with $0.3 \% \mathrm{H}_{2} \mathrm{O}_{2}$ in PBS (pH 7.4) $0.1 \%$ Triton X-100 for $45 \mathrm{~min}$ and non-specific antibody binding was blocked with normal horse serum in PBS ( $\mathrm{pH}$ 7.4) $0.1 \%$ Triton $\mathrm{X}-100$ for $60 \mathrm{~min}$. Sections were incubated $48 \mathrm{~h}$ at $4{ }^{\circ} \mathrm{C}$ with the rabbit primary antibody: anti-ChAT (1/1000) (Chemicon) and then with donkey anti-rabbit biotinylated antibody (1/200, Jackson ImmunoResearch) and visualized with the ABC diaminobenzidine reaction.

$D_{2} R$ cKO in $D_{2} R-M S N$, cholinergic, and dopaminergic neurons. cKO mice were generated by crossing homologous $\operatorname{Drd} 2^{\text {LoxP/LoxP }}$ with heterologous specific Cre $+1-$ driver mice and the resulting double heterologous $C r e^{-/+}:: D r d 2^{W t / / L o x P}$ are crossed with heterologous Drd2 $2^{\text {Wt//LoxP }}$. This crossing gives the cKO Cre ${ }^{+/-}:$:
$\operatorname{Drd} 2^{\operatorname{LoxP} / L o x P}$, the Cre driver control Cre ${ }^{+/-}:: \operatorname{Drd} 2^{W t / W t}$ and the floxed gene control Cre ${ }^{-/-}:: D r d 2^{\text {LoxP/LoxP }}$. The Cre-mediated deletion of Drd2 in $\mathrm{D}_{2} \mathrm{R}-\mathrm{MSN}$, striatal cholinergic interneurons, or dopaminergic neurons was obtained using mice expressing Cre-recombinase under the control of the Adora2a promoter (Adora2a-Cre mice ${ }^{47}$ ), ChAT promoter (ChAT-Cre; JAX SN6410), and Slc6a3 promoter (DAT-Cre mice), respectively.

Selective $\mathbf{D}_{\mathbf{2}} \mathbf{R}$ cKO in neocortical pyramidal cells of layer $\mathbf{5}$. We stereotaxically injected, in somatosensory cortex layer 5, AAV-cre-GFP (AAV1.CMV.HI.eGFPCre.WPRE.SV40, UPENN Core) in C57BL/6 $D_{2} R^{\text {LoxP/LoxP }}$ mice leading to selective $\mathrm{D}_{2} \mathrm{R}-\mathrm{KO}$ mice in corticostriatal cells; C57BL/6 $D_{2} R^{\text {LoxP/LoxP }}$ mice injected with AAV-GFP (AAV1.CMV.PI.eGFP.WPRE.bGH, UPENN Core) were used as the corresponding control. AAV-GFP and AAV-cre-GFP were stereotaxically injected at $0.1 \mu \mathrm{l} / \mathrm{min}$ with a blunt needle in four (bilaterally) sides of the somatosensory cortex with the following coordinates: anterior +1.2 and $+0.1 \mathrm{~mm}$, lateral \pm 3.5 and $\pm 3.8 \mathrm{~mm}$, ventral $-3.2 \mathrm{~mm}$. Electrophysiological recordings were performed from 15-30 days after viral injection.

$\mathbf{D}_{\mathbf{2}} \mathbf{R}$ immunostaining. $D_{2} R^{\operatorname{LoxP} / \operatorname{Lox} P}$ mice were anesthetized and perfused transcardially with $0.01 \mathrm{M}$ PBS, followed by $4 \%$ paraformaldehyde for tissue fixation. Brains were dissected, dehydrated in $20-30 \%$ sucrose solutions and frozen. Coronal sections of $16 \mu \mathrm{m}$ thickness were cut through the striatum with a freezing microtome (Leica). Non-specific antibody binding was blocked with normal horse serum in PBS (pH 7.4) $0.1 \%$ Triton X-100 for $60 \mathrm{~min}$. Sections were incubated $24 \mathrm{~h}$ at $4{ }^{\circ} \mathrm{C}$ with the rabbit primary antibody: anti- $\mathrm{D}_{2} \mathrm{R}(1 / 1000)$ (Frontier Science) and then with donkey anti-rabbit Alexa594 (1/200, Jackson ImmunoResearch).

SNc or MFB 6-OHDA-lesioned rats and L-DOPA treatment. 6-OHDA-lesioned animals: Sprague-Dawley rats weighing 125-150 g (Charles River Laboratories, L'Arbresle, France) were anesthetized with sodium pentobarbital $(30 \mathrm{mg} / \mathrm{kg}$ i.p. Ceva Sante Animale, Libourne, France) supplemented by injections of ketamine $(27.5 \mathrm{mg} / \mathrm{kg}$, im; Imalgène, Merial, Lyon, France). Thirty minutes before the injection of 6-OHDA (or vehicle in sham-operated animals), all animals received a bolus of desipramine ( $25 \mathrm{mg} / \mathrm{kg}$, i.p.; Sigma-Aldrich) to prevent neurotoxininduced damage of noradrenergic neurons. Animals were fixed in a stereotaxic frame (Kopf Instruments, Tujunga, CA, USA). Body temperature was maintained at $36.5^{\circ} \mathrm{C}$ with a homeothermic blanket (Harvard Apparatus, Kent, UK). A small craniotomy was made unilaterally (left side) over the SNc or MFB and the overlying dura mater was removed. For the Parkinson's disease model and for the removal of the nigrostriatal dopaminergic cells, an unilateral 6-OHDA lesion of the SNc and MFB were performed, respectively. A single stereotaxic injection of 6-OHDA (or of vehicle in the sham-operated animals) was delivered into the SNc on the left side (stereotaxic coordinates anteriority from the interaural line (A): 3.7 $\mathrm{mm}$, laterality from the midline $(\mathrm{L}): 2.1 \mathrm{~mm}$, depth from the cortical surface $(\mathrm{H})$ : $-7.55 \mathrm{~mm}$ ). Concerning the MFB lesion, 6-OHDA was injected into the left MFB; stereotaxic coordinates anteriority from the bregma (A): $4.5 \mathrm{~mm}$, laterality from the midline (L): $1.2 \mathrm{~mm}$, depth from the cortical surface $(\mathrm{H}):-7.9 \mathrm{~mm}$. 6-OHDA (hydrochloride salt; Sigma) was dissolved immediately prior use in ice-cold $0.9 \% \mathrm{w} / \mathrm{v} \mathrm{NaCl}$ solution containing $0.01 \% \mathrm{w} / \mathrm{v}$ ascorbic acid to a final concentration of $2.5 \mathrm{mg} / \mathrm{ml}$. $4.0 \mu \mathrm{l}$ of this 6 -OHDA solution (or vehicle) was injected at a rate of $16 \mu \mathrm{l} / \mathrm{h}$ via a steel cannula $(0.25 \mathrm{~mm}$ outside diameter $)$ attached to a $10 \mu \mathrm{l}$ Hamilton microsyringe (Cole-Parmer, London, UK) controlled by an electrical pump (KDS100; KD Scientific, Holliston, MA). After surgery, animals received an intramuscular injection of gentamicin to prevent bacterial infection $(3 \mathrm{mg} / \mathrm{kg}$, im; Gentalline, Schering-Plough, Levallois-Perret, France).

Chronic L-DOPA treatment: Two weeks after 6-OHDA injection, rats were splitted into two groups which received i.p. injection twice-daily for 10 days of either L-DOPA $(10 \mathrm{mg} / \mathrm{kg})$ with benzerazide $(7.5 \mathrm{mg} / \mathrm{kg})$ or saline. The shamoperated animals were also subjected to L-DOPA or saline injections with the same schedule. Animals received the last injection of L-DOPA or saline 30-60 min before being sacrified for ex vivo experiments.

TH immunostaining: The severity of the 6-OHDA lesions was quantified by striatal $\mathrm{TH}$ immunostaining. Brain slices were incubated in $0.1 \% \mathrm{H}_{2} \mathrm{O}_{2}$ and $10 \%$ methanol in PBS (15 min) and then in a 1/500 dilution of mouse anti-TH monoclonal antibody (MAB318; Merk-Millipore) overnight at $4{ }^{\circ} \mathrm{C}$. Biotin-goat anti-mouse secondary antibody (Life Technology-Invitrogen, Villebon-sur-Yvette, France) was incubated at a dilution of $1 / 500$ for $2.4 \mathrm{~h}$ at room temperature and visualized using with avidin-biotin complex (ABC Elite standard, PK-4000, Vector Laboratories, Burlingame, CA, USA) and DAB detection kit (Vector Laboratories).

Mathematical model. We used the mathematical model of corticostriatal synaptic plasticity ${ }^{13}$ with two additional parameters related to presynaptic plasticity (see below). This model implements a single dendritic spine and a soma as a single isopotential electrical compartment. Electrical activation is coupled to a detailed description of the postsynaptic signaling pathways from calcium currents to the activation of CaMKIIa and production of eCBs. Ordinary differential equations based on classical mass-action law formalisms are then used to express the 
corresponding reaction kinetics. Plasticity outcome is determined by $W_{\text {total }}$ that has pre-and postsynaptic components and was computed as $W_{\text {total }}=W_{\text {pre }} \cdot W_{\text {post }}$.

We modeled the glutamate concentration in the synaptic cleft, $G(t)$, as a train of exponentially-decaying impulses triggered by presynaptic stimuli at time $t_{\mathrm{pre}_{i}}$ :

$$
G(t)=G_{\max } \sum_{i} \exp \left(-\frac{t-t_{p_{i}}}{\tau_{G}}\right) \Theta\left(t-t_{p r e_{i}}\right)
$$

where $G_{\max }$ is the peak glutamate concentrations and $\tau_{G}$ is the glutamate clearance rate. On the postsynaptic side, action currents resulting from postsynaptic stimulations were modeled according to:

$$
I_{\text {action }}(t)=-D C_{\max } \sum_{\mathrm{i}} R\left(t, t_{\text {post }_{i}}, D C_{\text {dur }}\right)-A P_{\max } \sum_{\mathrm{i}} \Theta\left(t-\delta-t_{\text {post }_{i}}\right) \exp \left(\frac{-t+\delta+t_{\text {post }_{i}}}{\tau_{\mathrm{bAP}}}\right)
$$

where the rectangle function $R(t, a, l)=\Theta(t-a)-\Theta(t-a-l), D C_{\max }$ and $D C_{\mathrm{dur}}$ are the amplitude and duration of step-current injected in the postsynaptic soma, $A P_{\max }$ is the amplitude of the action current producing the back-propagating action potential (bAP); $\tau_{\mathrm{bAP}}$ is the timescale for bAP decay and $\delta$ accounts for the time elapsed between the onset of the postsynaptic step current and the action potential it triggers ( $\sim 3 \mathrm{~ms}$ in MSNs). We modeled the electrical response to these stimulations in a postsynaptic element considered as a single isopotential compartment with AMPAR, NMDAR, VSCC, and TRPV1 conductances:

$$
\begin{aligned}
C_{\mathrm{m}} \frac{d V}{d t}=- & g_{L}\left(V-V_{\mathrm{L}}\right)-I_{\mathrm{AMPAR}}(V, G(t))-I_{\mathrm{NMDAR}}(V, G(t)) \\
& -I_{\mathrm{VSCC}}(V)-I_{\mathrm{TRPV} 1}(V, A E A)-I_{\text {action }}(t)
\end{aligned}
$$

where $V$ is membrane potential; $g_{L}$ and $V_{L}$ are leak conductance and reversal potential, respectively, and AEA stands for anandamide. For a detailed exposition of the models used for those currents, see refs. ${ }^{12,13}$.

The dynamics of free cytosolic calcium $C$ was computed according to:

$$
T_{\mathrm{C}}(C) \frac{d C}{d t}=J_{I P_{3} R}-J_{\mathrm{SERCA}}+J_{\text {leak }}+J_{\mathrm{NMDAR}}+J_{\mathrm{VSCC}}+J_{\mathrm{TRPV} 1}-\frac{C-C_{\mathrm{b}}}{\tau_{C_{\mathrm{b}}}}
$$

where the fluxes $J_{\mathrm{IP} 3 \mathrm{R}}, J_{\mathrm{SERCA}}, J_{\text {leak }}$ from and to the ER in the Calcium-Induced Calcium Release (CICR) system were taken from the model of De Pittà et al. ${ }^{73}$ (see also ref. ${ }^{12}$ ) and $J_{\text {NMDAR }}, J_{\mathrm{VSCC}}$, and $J_{\mathrm{TRPV} 1}$ are the calcium fluxes from the plasma membrane channels (Eq. (3)). $C_{b}$ is the basal cytosolic calcium level resulting from equilibration with calcium diffusion out of the cell and $\tau_{\mathrm{C}_{\mathrm{b}}}$ is the corresponding timescale. $T_{C}(C)$ is a time scaling factor accounting for the presence of endogenous calcium buffers. See Cui et al. ${ }^{12}$ for details.

$W_{\text {post }}$ was based on the activation by calcium of calmodulin and CaMKII and the regulation of this system by PKA, calcineurin and protein phosphatase 1 (PP1). The model of Cui et al. ${ }^{12}$ for this subsection is based on the biophysical model proposed in Graupner and Brunel ${ }^{74}$, that expresses the calcium-dependent kinetics of the protein complexes and enzymes along this pathway, including the calciumdependent kinetics of CaMKII* the activated form of CaMKII, that was assumed to set postsynaptic plasticity according to:

$$
W_{\text {post }}=1+3.5 \frac{\mathrm{CaMKII}^{*}}{\mathrm{CaMKII}}
$$

where CaMKII ${ }_{\max }^{*}$ is the maximal concentration of activate (phosphorylated) CaMKII.

Our model also accounts for the biochemical pathways leading to the production of the endocannabinoids 2-arachidonoylglycerol (2-AG) and AEA, and their subsequent $\mathrm{CB}_{1} \mathrm{R}$ activation. We modeled $\mathrm{CB}_{1} \mathrm{R}$ activation by 2-AG and AEA using a simple three-state kinetic model: open $\left(x_{\mathrm{CB} 1 \mathrm{R}}\right)$, desensitized $\left(d_{\mathrm{CB} 1 \mathrm{R}}\right)$ and inactivated $\left(i_{\mathrm{CB} 1 \mathrm{R}}\right)$.

$$
\begin{gathered}
\frac{d x_{C B 1 R}}{d t}=\alpha_{C B 1 R} e C B\left(1-d_{C B 1 R}-x_{C B 1 R}\right)-\left(\beta_{C B 1 R}+\gamma_{C B 1 R}\right) x_{C B 1 R} \\
\frac{d d_{C B 1 R}}{d t}=-\varepsilon_{C B 1 R} d_{C B 1 R}+\gamma_{C B 1 R} x_{C B 1 R}
\end{gathered}
$$

where $e C B=2-A G+0.10 A E A$ accounts for the act that AEA is a partial agonist of $\mathrm{CB}_{1} \mathrm{R}$.

In the original model ${ }^{13}, \mathrm{CB}_{1} \mathrm{R}$ activation controls presynaptic plasticity via the $\mathrm{CB}_{1} \mathrm{R}$-triggered $\mathrm{G}_{\mathrm{i} / 0}-\mathrm{GPCR}$ pathway. Here, we add the assumption that the effects of $\mathrm{D}_{2} \mathrm{R}$ and $\mathrm{CB}_{1} \mathrm{R}$ on $W_{\text {pre }}$ are cumulative. We account here for the resulting synergistic effects by combining both effects linearly in the variable $y_{G}$ that controls $W_{\text {pre: }}$

$$
y_{G}=k_{\mathrm{CB} 1 \mathrm{R}} \cdot x_{\mathrm{CB} 1 \mathrm{R}}+\gamma_{\mathrm{D} 2 \mathrm{R}} \cdot \mathrm{DA}+\gamma_{\mathrm{other}}
$$

where $x_{\mathrm{CB} 1 \mathrm{R}}$ is the fraction of activated (non-desensitized) $\mathrm{CB}_{1} \mathrm{R}, k_{\mathrm{CB} 1 \mathrm{R}}$ quantifies the effect of $\mathrm{CB}_{1} \mathrm{R}$ activation, $\mathrm{DA}$ is the concentration of (tonic) dopamine, $\gamma_{\mathrm{D} 2 \mathrm{R}}$ quantifies the effect of $D_{2} R$ activation and $\gamma_{\text {other }}$ summarizes the effects of other $G_{i /}$ ${ }^{-}$-GPCR pathways (beyond $\mathrm{CB}_{1} \mathrm{R}$ and $\mathrm{D}_{2} \mathrm{R}$ ). Note that $y_{\mathrm{G}}$ was actually referred to as $y_{\mathrm{CB} 1 \mathrm{R}}$ in ref. ${ }^{13}$ whereas the term $\gamma_{\mathrm{D} 2 \mathrm{R}}$. DA $+\gamma_{\text {other }}$ was lumped in a constant $D_{1}$.
Finally, we assume that $y_{G}$ controls $W_{\text {pre }}$, which we express in the model via two functions $\Omega\left(y_{G}\right)$ and $\tau_{W_{\text {pre }}}\left(y_{G}\right)$ :

$$
\begin{gathered}
\frac{d W_{\text {pre }}}{d t}=\frac{\Omega\left(y_{G}\right)-W_{\text {pre }}}{\tau_{W_{\text {pre }}\left(y_{G}\right)},} \\
\tau_{W_{\text {pre }}}\left(y_{G}\right)=\frac{P_{1}}{P_{2}^{P_{3}}+\left(y_{G}+P_{5}\right)^{P_{3}}}+P_{4}, \\
\Omega\left(y_{G}\right)= \begin{cases}1+A_{L T P}, & \text { if } \theta_{\mathrm{LTD}}^{\text {start }}<y_{G}<\theta_{\mathrm{LTD}}^{\text {stop }}, \\
1-A_{L T D}, & \text { if } y_{G}>\theta_{\mathrm{LTP}}^{\text {start }}, \\
1, & \text { otherwise. }\end{cases}
\end{gathered}
$$

where $A_{L T P}, A_{L T D}, P_{1-5}$ are positive constants; $\theta_{\mathrm{LTD}}^{\text {start }}, \theta_{\mathrm{LTD}}^{\text {stop }}$, and $\theta_{\mathrm{LTP}}^{\text {start }}$ are plasticity thresholds. Here, $\Omega$ sets the steady-state presynaptic weight, while $1 / \tau_{W_{p r e}}$ sets the rate at which this steady-state is approached. $\Omega$ decreases (LTD) when $y_{G}$ is in between the two threshold values, $\theta_{\mathrm{LTD}}^{\text {start }}$ and $\theta_{\mathrm{LTD}}^{\mathrm{stop}}$ whereas $\Omega$ rises (LTP) if $y_{G}$ becomes larger than $\theta_{\mathrm{LTP}}^{\text {start }}$ (dashed line in Fig. 8c). Therefore, $W_{\text {pre }}$ depends on $y_{G}$ and $y_{G}$ indirectly depends on cytoplasmic calcium, thus on spike timing. As a result, $W_{\text {pre }}$ depends on spike timing in the model.

In the present study, we used $k_{\mathrm{CB1R}}=2400 ; \gamma_{\mathrm{D} 2 \mathrm{R}}=0.84, \mathrm{DA}=0.01 \mu \mathrm{M}, \gamma_{\text {other }}$ $=12.6 \cdot 10^{-3} ; A_{\mathrm{LTD}}=1.82, A_{\mathrm{LTP}}=24, \theta_{\mathrm{LTD}}^{\text {stop }}=0.053$ and $\theta_{\mathrm{LTP}}^{\text {start }}=0.085$. For all other parameters, we used the same values as in ref. ${ }^{13}$

\section{Data availability}

All relevant data are available from the authors. The mathematical model can be downloaded from https://senselab.med.yale.edu/modeldb/, accession \#187605.

Received: 25 May 2016 Accepted: 30 August 2018 Published online: 08 October 2018

\section{References}

1. Castillo, P. E., Younts, T. J., Chávez, A. E. \& Hashimotodani, Y. Endocannabinoid signaling and synaptic function. Neuron 76, 70-81 (2012).

2. Araque, A., Castillo, P. E., Manzoni, O. J. \& Tonini, R. Synaptic functions of endocannabinoid signaling in health and disease. Neuropharmacology 124, 13-24 (2017).

3. Cachope, R., Mackie, K., Triller, A., O’Brien, J. \& Pereda, A. E. Potentiation of electrical and chemical synaptic transmission mediated by endocannabinoids. Neuron 56, 1034-1047 (2007).

4. Zhu, P. J. \& Lovinger, D. M. Persistent synaptic activity produces long-lasting enhancement of endocannabinoid modulation and alters long-term synaptic plasticity. J. Neurophysiol. 97, 4386-4389 (2007).

5. Xu, J.-Y., Zhang, J. \& Chen, C. Long-lasting potentiation of hippocampal synaptic transmission by direct cortical input is mediated via endocannabinoids. J. Physiol. 590, 2305-2315 (2012).

6. Lin, Q.-S. et al. Hippocampal endocannabinoids play an important role in induction of long-term potentiation and regulation of contextual fear memory formation. Brain Res. Bull. 86, 139-145 (2011).

7. Carlson, G., Wang, Y. \& Alger, B. E. Endocannabinoids facilitate the induction of LTP in the hippocampus. Nat. Neurosci. 5, 723-724 (2002).

8. Navarrete, M. \& Araque, A. Endocannabinoids potentiate synaptic transmission through stimulation of astrocytes. Neuron 68, 113-126 (2010).

9. Wang, W. et al. A primary cortical input to hippocampus expresses a pathway-specific and endocannabinoid-dependent form of long-term potentiation. eNeuro 3, 10049-10053 (2016).

10. Wang, W. et al. Atypical endocannabinoid signaling initiates a new form of memory-related plasticity at a cortical input to hippocampus. Cereb. Cortex 28, 2253-2266 (2018)

11. Cui, Y., Perez, S. \& Venance, L. Endocannabinoid-LTP mediated by CB1 and TRPV1 receptors encodes for limited occurrences of coincident activity in neocortex. Front. Cell. Neurosci. 12, 182 (2018).

12. Cui, Y. et al. Endocannabinoids mediate bidirectional striatal spike-timing dependent plasticity. J. Physiol. 593, 2833-2849 (2015).

13. Cui, Y. et al. Endocannabinoid dynamics gate spike-timing dependent depression and potentiation. eLife 5, e13185 (2016).

14. Yin, H. H. et al. Dynamic reorganization of striatal circuits during the acquisition and consolidation of a skill. Nat. Neurosci. 12, 333-341 (2009).

15. Koralek, A. C., Jin, X., Long, J. D. II, Costa, R. M. \& Carmena, J. M. Corticostriatal plasticity is necessary for learning intentional neuroprosthetic skills. Nature 483, 331-335 (2012). 
16. Shan, Q., Ge, M., Christie, M. J. \& Balleine, B. W. The acquisition of goaldirected actions generates opposing plasticity in direct and indirect pathways in dorsomedial striatum. J. Neurosci. 34, 9196-9201 (2014).

17. Rothwell, P. E. et al. Input- and output-specific regulation of serial order performance by corticostriatal circuits. Neuron 88, 345-356 (2015).

18. Hawes, S. L. et al. Multimodal plasticity in dorsal striatum while learning a lateralized navigation task. J. Neurosci. 35, 10535-10549 (2015).

19. Xiong, Q., Znamenskiy, P. \& Zador, A. M. Selective corticostriatal plasticity during acquisition of an auditory discrimination task. Nature 521, 348-351 (2015).

20. Pasupathy, A. \& Miller, E. K. Different time courses of learning-related activity in the prefrontal cortex and striatum. Nature 433, 873-876 (2005).

21. Koralek, A., Costa, R. \& Carmena, J. Temporally precise cell-specific coherence develops in corticostriatal networks during learning. Neuron 79, 865-872 (2013).

22. Schultz, W. Neuronal reward and decision signals: from theories to data. Physiol. Rev. 95, 853-951 (2015).

23. Surmeier, D. J., Graves, S. M. \& Shen, W. Dopaminergic modulation of striatal networks in health and Parkinson's disease. Curr. Opin. Neurobiol. 29, 109-117 (2014).

24. Mathur, B. N. \& Lovinger, D. M. Endocannabinoid-dopamine interactions in striatal synaptic plasticity. Front. Pharmacol. 3, 66 (2012).

25. Di Filippo, M. et al. Short-term and long-term plasticity at corticostriatal synapses: implications for learning and memory. Behav. Brain Res. 199, 108-118 (2009).

26. Fino, E. \& Venance, L. Spike-timing dependent plasticity in the striatum. Front. Synaptic Neurosci. 2, 6 (2010).

27. Feldman, D. E. The spike-timing dependence of plasticity. Neuron 75, 556-571 (2012).

28. Shen, W., Flajolet, M., Greengard, P. \& Surmeier, D. J. Dichotomous dopaminergic control of striatal synaptic plasticity. Science 321, 848-851 (2008).

29. Pawlak, V. \& Kerr, J. N. D. Dopamine receptor activation is required for corticostriatal spike-timing-dependent plasticity. J. Neurosci. 28, 2435-2446 (2008).

30. Fino, E., Glowinski, J. \& Venance, L. Bidirectional activity-dependent plasticity at corticostriatal synapses. J. Neurosci. 25, 11279-11287 (2005).

31. Fino, E. et al. Distinct coincidence detectors govern the corticostriatal spike timing-dependent plasticity. J. Physiol. 588, 3045-3062 (2010).

32. Paillé, V. et al. GABAergic circuits control spike-timing-dependent plasticity. J. Neurosci. 33, 9353-9363 (2013).

33. Tritsch, N. X. \& Sabatini, B. L. Dopaminergic modulation of synaptic transmission in cortex and striatum. Neuron 76, 33-50 (2012).

34. Zhuang, X., Mazzoni, P. \& Kang, U. J. The role of neuroplasticity in dopaminergic therapy for Parkinson disease. Nat. Rev. Neurol. 9, 248-256 (2013).

35. Valtcheva, S. et al. Developmental control of spike-timing-dependent plasticity by tonic GABAergic signaling in striatum. Neuropharmacology 121, 261-277 (2017).

36. Schulz, J. M., Redgrave, P. \& Reynolds, J. N. J. Cortico-striatal spike-timing dependent plasticity after activation of subcortical pathways. Front. Synaptic Neurosci. 2, 23 (2010).

37. Fino, E., Deniau, J.-M. \& Venance, L. Brief subthreshold events can act as Hebbian signals for long-term plasticity. PloS One 4, e6557 (2009).

38. Strafella, A. P., Paus, T., Fraraccio, M. \& Dagher, A. Striatal dopamine release induced by repetitive transcranial magnetic stimulation of the human motor cortex. Brain 126, 2609-2615 (2003).

39. Juranyi, Z., Zigmond, M. J. \& Harsing, L. G. [3H]Dopamine release in striatum in response to cortical stimulation in a corticostriatal slice preparation. J. Neurosci. Methods 126, 57-67 (2003).

40. Quiroz, C. et al. Key modulatory role of presynaptic adenosine A 2A receptors in cortical neurotransmission to the striatal direct pathway. ScientificWorldJournal 9, 1321-1344 (2009).

41. Mahn, M., Prigge, M., Ron, S., Levy, R. \& Yizhar, O. Biophysical constraints of optogenetic inhibition at presynaptic terminals. Nat. Neurosci. 19, 554-556 (2016).

42. Atwood, B. K., Lovinger, D. M. \& Mathur, B. N. Presynaptic long-term depression mediated by Gi/o-coupled receptors. Trends Neurosci. 37, 663-673 (2014).

43. Calabresi, P., Picconi, B., Tozzi, A., Ghiglieri, V. \& Di Filippo, M. Direct and indirect pathways of basal ganglia: a critical reappraisal. Nat. Neurosci. 17, 1022-1030 (2014).

44. Hersch, S. M. et al. Electron microscopic analysis of D1 and D2 dopamine receptor proteins in the dorsal striatum and their synaptic relationships with motor corticostriatal afferents. J. Neurosci. 15, 5222-5237 (1995).

45. Sesack, S. R., Aoki, C. \& Pickel, V. M. Ultrastructural localization of D2 receptor-like immunoreactivity in midbrain dopamine neurons and their striatal targets. J. Neurosci. 14, 88-106 (1994).
46. Wang, H. \& Pickel, V. M. Dopamine D2 receptors are present in prefrontal cortical afferents and their targets in patches of the rat caudate-putamen nucleus. J. Comp. Neurol. 442, 392-404 (2002).

47. Bamford, N. S. et al. Dopamine modulates release from corticostriatal terminals. J. Neurosci. 24, 9541-9552 (2004).

48. Durieux, P. F. et al. D2R striatopallidal neurons inhibit both locomotor and drug reward processes. Nat. Neurosci. 12, 393-395 (2009).

49. Sjöström, P. J., Turrigiano, G. G. \& Nelson, S. B. Rate, timing, and cooperativity jointly determine cortical synaptic plasticity. Neuron $\mathbf{3 2}$ 1149-1164 (2001).

50. Cui, Y., Prokin, I., Mendes, A., Berry, H. \& Venance, L. Robustness of STDP to spike timing jitter. Sci. Rep. 8, 8139 (2018).

51. Volkow, N. D., Fowler, J. S., Wang, G. J., Baler, R. \& Telang, F. Imaging dopamine's role in drug abuse and addiction. Neuropharmacology 56, 3-8 (2009).

52. Barnes, T. D. et al. Advance cueing produces enhanced action-boundary patterns of spike activity in the sensorimotor striatum. J. Neurophysiol. 105, 1861-1878 (2011)

53. Goubard, V., Fino, E. \& Venance, L. Contribution of astrocytic glutamate and GABA uptake to corticostriatal information processing. J. Physiol. 589, 2301-2319 (2011).

54. Russell, M., Winitz, S. \& Johnson, G. L. Acetylcholine muscarinic m1 receptor regulation of cyclic AMP synthesis controls growth factor stimulation of Raf activity. Mol. Cell. Biol. 14, 2343-2351 (1994).

55. Wang, Z. et al. Dopaminergic control of corticostriatal long-term synaptic depression in medium spiny neurons is mediated by cholinergic interneurons. Neuron 50, 443-452 (2006).

56. Tozzi, A. et al. The distinct role of medium spiny neurons and cholinergic interneurons in the D2/A2A receptor interaction in the striatum: implications for Parkinson's disease. J. Neurosci. 31, 1850-1862 (2011).

57. Kreitzer, A. C. \& Malenka, R. C. Endocannabinoid-mediated rescue of striatal LTD and motor deficits in Parkinson's disease models. Nature 445, 643-647 (2007).

58. Lerner, T. \& Kreitzer, A. RGS4 is required for dopaminergic control of striatal LTD and susceptibility to Parkinsonian motor deficits. Neuron 73, 347-359 (2012).

59. Mateo, Y. et al. Endocannabinoid actions on cortical terminals orchestrate local modulation of dopamine release in the nucleus accumbens. Neuron 96 1112-1126 (2017).

60. Fisher, S. D. et al. Reinforcement determines the timing dependence of corticostriatal synaptic plasticity in vivo. Nat. Commun. 8, 334 (2017).

61. Shindou, T., Shindou, M., Watanabe, S. \& Wickens, J. A silent eligibility trace enables dopamine-dependent synaptic plasticity for reinforcement learning in the mouse striatum. Eur. J. Neurosci. https://doi.org/10.1111/ejn.13921 (2018).

62. Yagishita, S. et al. A critical time window for dopamine actions on the structural plasticity of dendritic spines. Science 345, 1616-1620 (2014).

63. Graupner, M. \& Brunel, N. Calcium-based plasticity model explains sensitivity of synaptic changes to spike pattern, rate, and dendritic location. Proc. Natl Acad. Sci. U.S.A. 109, 3991-3996 (2012).

64. Shouval, H. Z., Bear, M. F. \& Cooper, L. N. A unified model of NMDA receptor-dependent bidirectional synaptic plasticity. Proc. Natl Acad. Sci. U.S. A. 99, 10831-10836 (2002).

65. Rice, M. E., Patel, J. C. \& Cragg, S. J. Review dopamine release in the basal ganglia. Neuroscience 198, 112-137 (2011).

66. Marcott, P. F., Mamaligas, A. A. \& Ford, C. P. Phasic dopamine release drives rapid activation of striatal d2-receptors. Neuron 84, 164-176 (2014).

67. Brzosko, Z., Schultz, W. \& Paulsen, O. Retroactive modulation of spike timing-dependent plasticity by dopamine. eLife 4, e09685 (2015).

68. Planert, H., Berger, T. K. \& Silberberg, G. Membrane properties of striatal direct and indirect pathway neurons in mouse and rat slices and their modulation by dopamine. PloS One 8, e57054 (2013).

69. Day, M., Wokosin, D., Plotkin, J. L., Tian, X. \& Surmeier, D. J. Differential excitability and modulation of striatal medium spiny neuron dendrites. J. Neurosci. 28, 11603-11614 (2008).

70. Cazorla, M., Kang, U. J. \& Kellendonk, C. Balancing the basal ganglia circuitry: a possible new role for dopamine D2 receptors in health and disease. Mov. Disord. 30, 895-903 (2015).

71. Buch, T. et al. A Cre-inducible diphtheria toxin receptor mediates cell lineage ablation after toxin administration. Nat. Methods 2, 419-426 (2005).

72. Bello, E. P. et al. Cocaine supersensitivity and enhanced motivation for reward in mice lacking dopamine D2 autoreceptors. Nat. Neurosci. 14, 1033-1038 (2011).

73. De Pittà, M., Goldberg, M., Volman, V., Berry, H. \& Ben-Jacob, E. Glutamate regulation of calcium and IP3 oscillating and pulsating dynamics in astrocytes. J. Biol. Phys. 35, 383-411 (2009).

74. Graupner, M. \& Brunel, N. STDP in a bistable synapse model based on CaMKII and associated signaling pathways. PLoS Comput. Biol. 3, 2299-2323 (2007). 


\section{Acknowledgements}

We thank M. Rubinstein and E. Valjent for the Drd2 floxed mice. We thank the LV lab members for helpful suggestions and critical comments. This work was supported by grants from INSERM, Collège de France, the Agence Nationale pour la Recherche (ANR-CRCNS Dopaciumcity), France Parkinson, INRIA, CNRS, Fondation de France, FRS-FNRS, and the Ecole des Neurosciences de Paris. B.D. and A.C. are research Fellow of the FRS-FNRS (Belgium). A.K.E. is a Senior Research Associate of the FRS-FNRS and a WELBIO investigator and is supported by FRS-FNRS (Belgium), FER from ULB, Action de Recherche Concertée from the CFWB.

\section{Author contributions}

H.X. and L.V. designed the study; H.X., S.P., and Y.C. performed electrophysiological experiments; H.X., S.P., and L.V. performed analysis; H.B. carried out the conception and the design of the mathematical model; H.B. and I.P. performed the acquisition and analysis of data from the mathematical model; B.D. and A.K.E. generated the Adora2a-Cre and produced the $A_{2 A^{-}} C r e^{+/-}:: i D T R^{+/-}:: D r d 1 a-G F P^{+/-}$, ChAT-cre ${ }^{+/-}::$ $i D T R^{+/-}:: D r d 1 a-G F P^{+/-}$triple transgenic mice, the ChAT-Cre ${ }^{+/-}:: i D T R^{+/-}$double transgenic mice and performed stereotaxic injection of DT; B.D. did the immunohistochemistry and in situ hybridization; A.K.E. and A.C. generated $\mathrm{D}_{2} \mathrm{R}$-cKO mice

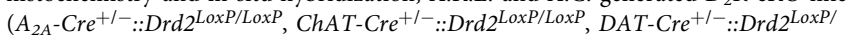
LoxP mice), and the corresponding control mice (the Cre driver controls: $\mathrm{A}_{2 \mathrm{~A}^{-}} \mathrm{Cre}^{+/-}::$ $\mathrm{Drd} 2^{W t / W t}, \mathrm{ChAT}-\mathrm{Cr} e^{+/-}:: \mathrm{Drd} 2^{W t / W t}$ and $\mathrm{DAT}-\mathrm{Cr} e^{+/-}:: \mathrm{Drd} 2^{W t / W t}$ mice; the floxed gene control: $C r e^{-/-}:: D r d 2^{\operatorname{Lox} P / L o x P}$ mice); A.C. performed stereotaxic injection of $A A V$-cre-GFP in $D_{2} R^{L o x P / L o x P}$ mice; B.D. and S.P. performed 6-OHDA lesions; H.X., H.B., and L.V. wrote the manuscript and all authors have edited and corrected the manuscript; L.V. supervised the whole study.

\section{Additional information}

Supplementary Information accompanies this paper at https://doi.org/10.1038/s41467018-06409-5.

Competing interests: The authors declare no competing interests.

Reprints and permission information is available online at http://npg.nature.com/ reprintsandpermissions/

Publisher's note: Springer Nature remains neutral with regard to jurisdictional claims in published maps and institutional affiliations.

(c) (i) Open Access This article is licensed under a Creative Commons Attribution 4.0 International License, which permits use, sharing, adaptation, distribution and reproduction in any medium or format, as long as you give appropriate credit to the original author(s) and the source, provide a link to the Creative Commons license, and indicate if changes were made. The images or other third party material in this article are included in the article's Creative Commons license, unless indicated otherwise in a credit line to the material. If material is not included in the article's Creative Commons license and your intended use is not permitted by statutory regulation or exceeds the permitted use, you will need to obtain permission directly from the copyright holder. To view a copy of this license, visit http://creativecommons.org/ licenses/by/4.0/.

(C) The Author(s) 2018 